\title{
Effects of Turbulent Reynolds Number on the Displacement Speed Statistics in the Thin Reaction Zones Regime of Turbulent Premixed Combustion
}

\author{
Nilanjan Chakraborty, ${ }^{1}$ Markus Klein, ${ }^{2}$ and R. S. Cant ${ }^{3}$ \\ ${ }^{1}$ Engineering Department, University of Liverpool, Brownlow Hill, Liverpool L69 3GH, UK \\ ${ }^{2}$ Technische Universitaet Darmstadt, Institut fuer Energie -und Kraftwerkstechnik, Petersenstraße 30, 64287 Darmstadt, Germany \\ ${ }^{3}$ Engineering Department, Cambridge University, Trumpington Street, Cambridge CB2 1PZ, UK
}

Correspondence should be addressed to Nilanjan Chakraborty, n.chakraborty@liverpool.ac.uk

Received 15 January 2011; Accepted 28 March 2011

Academic Editor: A. Lipatnikov

Copyright ( 2011 Nilanjan Chakraborty et al. This is an open access article distributed under the Creative Commons Attribution License, which permits unrestricted use, distribution, and reproduction in any medium, provided the original work is properly cited.

The effects of turbulent Reynolds number on the statistical behaviour of the displacement speed have been studied using three-dimensional Direct Numerical Simulation of statistically planar turbulent premixed flames. The probability of finding negative values of the displacement speed is found to increase with increasing turbulent Reynolds number when the Damköhler number is held constant. It has been shown that the statistical behaviour of the Surface Density Function, and its strain rate and curvature dependence, plays a key role in determining the response of the different components of displacement speed. Increasing the turbulent Reynolds number is shown to reduce the strength of the correlations between tangential strain rate and dilatation rate with curvature, although the qualitative nature of the correlations remains unaffected. The dependence of displacement speed on strain rate and curvature is found to weaken with increasing turbulent Reynolds number when either Damköhler or Karlovitz number is held constant, but the qualitative nature of the correlation remains unaltered. The implications of turbulent Reynolds number effects in the context of Flame Surface Density (FSD) modelling have also been addressed, with emphasis on the influence of displacement speed on the curvature and propagation terms in the FSD balance equation.

\section{Introduction}

Displacement speed $S_{d}$ is an important quantity for the modelling of turbulent premixed flames using both levelset [1] and flame surface density (FSD) [2] approaches. The displacement speed is defined as the speed at which the flame surface moves normal to itself with respect to an initially coincident material surface. Statistics of displacement speed for turbulent premixed flames have been studied extensively in previous work based on two-dimensional direct numerical simulations (DNS) with detailed chemistry [3-13] and on three-dimensional DNS with simplified chemistry [14-22]. These studies have looked at the effects of local strain rate and curvature effect on $S_{d}$ [13-24] and have included the influence of root-mean-square (rms) turbulent velocity fluctuation magnitude $u^{\prime}$ and Lewis number Le $[9,15,17,22]$ on these effects. Peters [1] showed using an order of magnitude analysis that the curvature dependence of displacement speed becomes the leading order contributor in the thin reaction zones regime, whereas the curvature dependence remains relatively weak in the corrugated flamelets regime. This observation was supported using DNS data by Chakraborty [21] who also studied the individual response of the reaction, normal displacement, and curvature components of displacement speed. However, the effects of turbulent Reynolds number on displacement speed have yet to be addressed in detail. Hence, in this paper, the influence of $\mathrm{Re}_{t}$ on the statistics of $S_{d}$ has been analysed based on a three-dimensional compressible DNS database of statistically planar turbulent 
premixed flames. The main objectives of this study are as follows:

(1) to demonstrate the influence of $\mathrm{Re}_{t}$ on the statistical distribution of $S_{d}$

(2) to demonstrate the influence of $\mathrm{Re}_{t}$ on the local curvature and strain rate dependencies of $S_{d}$

(3) to indicate the implications of the dependence of $S_{d}$ on $\mathrm{Re}_{t}$ in the context of flame surface density-based turbulent combustion modelling.

In order to address the above objectives, a series of DNS runs has been carried out for turbulent Reynolds numbers ranging from 20 to 100 . In one group of simulations, the change in $\mathrm{Re}_{t}$ is obtained by modifying the Karlovitz number Ka for a given value of Damköhler number $\mathrm{Da}$, whereas in the other group of simulations the change in turbulent Reynolds number is induced by modifying the Damköhler number for a given value of Karlovitz number.

The rest of the paper is organised as follows. The mathematical background and the numerical details relevant to this work are discussed in the next two sections of this paper. Following this, results will be presented and subsequently discussed. In the final section, the main findings are summarised and conclusions are drawn.

\section{Mathematical Background}

Direct numerical simulations (DNS) of turbulent reacting flows ideally should be carried out in three-dimensions with detailed chemistry. However, such simulations remain extremely expensive and are not yet feasible for parametric studies. Previous work has indicated that displacement speed statistics obtained from three-dimensional DNS with simplified chemistry [14-22] are in agreement with those obtained from two-dimensional DNS with complex chemistry [3-13]. In the present study, three-dimensional DNS with simplified chemistry is adopted in order to allow for an extensive parametric study without excessive computational cost. The chemical reaction is represented in terms of single step Arrhenius type chemistry, and the species field is uniquely represented by a reaction progress variable $c$, which may be defined based on a suitable product mass fraction $Y_{P}$ according to

$$
c=\frac{Y_{P}-Y_{P 0}}{Y_{P \infty}-Y_{P 0}},
$$

where subscripts 0 and $\infty$ are used to refer to quantities in unburned reactants and fully burned products, respectively. The transport equation for reaction progress variable is given by

$$
\rho \frac{\partial c}{\partial t}+\rho u_{j} \frac{\partial c}{\partial x_{j}}=\dot{w}+\frac{\partial}{\partial x_{j}}\left(\rho D \frac{\partial c}{\partial x_{j}}\right)
$$

where $\dot{w}$ is the chemical reaction rate, $\rho$ is the fluid density, and $D$ is the diffusivity of reaction progress variable. The transport equation for reaction progress variable can be written in kinematic form for a given isosurface defined by $c=c^{*}[6,7]$

$$
\rho \frac{\partial c}{\partial t}+\rho u_{j} \frac{\partial c}{\partial x_{j}}=\rho S_{d}|\nabla c|_{c=c^{*}}
$$

Comparing (2a) and (2b), the expression for the displacement speed of a given $c=c^{*}$ isosurface can be obtained as $[1,3-7]$

$$
S_{d}=\left.\frac{\dot{w}+\nabla \cdot(\rho D \nabla c)}{\rho|\nabla c|}\right|_{c=c^{*}} .
$$

It is often useful to decompose the displacement speed $S_{d}$ into three separate components [3-7]

$$
S_{d}=S_{r}+S_{n}+S_{t},
$$

where $S_{r}, S_{n}$, and $S_{t}$ are the reaction, normal diffusion, and tangential diffusion components which are given as

$$
\begin{gathered}
S_{r}=\left.\frac{\dot{w}}{\rho|\nabla c|}\right|_{c=c^{*}}, \quad S_{n}=\left.\frac{\vec{N} \cdot \nabla(\rho D \vec{N} \cdot \nabla c)}{\rho|\nabla c|}\right|_{c=c^{*}}, \\
S_{t}=-2 D \kappa_{m},
\end{gathered}
$$

where $\kappa_{m}$ is the arithmetic mean of the two principal curvatures, which is defined as

$$
\kappa_{m}=\frac{1}{2} \nabla \cdot \vec{N} .
$$

According to (6), flame surface that is curved convex to the unburned reactants has a positive curvature and vice versa. The definition of displacement speed and its components in (4)-(6) suggests that the statistics of the surface density function $(\operatorname{SDF}=|\nabla c|)[15,25-27]$ and curvature $\kappa_{m}$ are each likely to have a significant effect on the statistical behaviour of displacement speed. The transport equation for the SDF for an isosurface of $c$ is given by $[15,25-27]$

$$
\frac{\partial|\nabla c|}{\partial t}+\frac{\partial\left(u_{j}|\nabla c|\right)}{\partial x_{j}}=a_{T}|\nabla c|+2 S_{d} \kappa_{m}|\nabla c|-\frac{\partial\left(S_{d} N_{i}|\nabla c|\right)}{\partial x_{i}},
$$

where $a_{T}$ is the tangential strain rate given by

$$
a_{T}=\left[\left(\delta_{i j}-N_{i} N_{j}\right) \frac{\partial u_{i}}{\partial x_{j}}\right]_{c=c^{*}} .
$$

It is evident from (7) and (8) that tangential strain rate is likely to affect the statistical behaviour of $|\nabla c|$, which in turn can affect the local curvature and strain rate dependence of $S_{r}$ and $S_{n}$. It is worth noting that in some previous studies [312], the statistics of the density-weighted displacement speed $S_{d}^{*}=\rho S_{d} / \rho_{0}$ were analysed instead of the statistics of $S_{d}$ itself. For low Mach number unity Lewis number flames, $S_{d}^{*}$ and $S_{d}$ are related by $S_{d}^{*}=S_{d}\left(1+\tau c^{*}\right)$ for a given isosurface of $c$, and thus the statistics of $S_{d}^{*}$ are similar to those of $S_{d}$. For this reason, and also since $S_{d}$ appears explicitly in the SDF and 
FSD transport equations [2, 15, 25-27], the statistics of $S_{d}$ are addressed in the present work. The local strain rate and curvature dependence of $S_{d}$ and its components for different values of turbulent Reynolds number will be discussed in Section 4 of this paper.

It is essential for the purposes of the present study to note the relationship between the turbulent Reynolds number and both the Damköhler and Karlovitz numbers. The turbulent Reynolds number is defined as $\operatorname{Re}_{t}=\rho_{0} u^{\prime} l / \mu$, where $\rho_{0}$ is the density and $\mu$ is the dynamic viscosity of the unburned reactants, and $l$ is the integral length scale of the turbulence. For unity Lewis number flames, the turbulent Reynolds number can be scaled as [1]

$$
\begin{aligned}
\mathrm{Re}_{t} & \sim \mathrm{Da}^{2} \mathrm{Ka}^{2} \sim\left(\frac{u^{\prime}}{S_{L}}\right)^{2} \mathrm{Da} \sim \frac{\left(u^{\prime} / S_{L}\right)^{4}}{\mathrm{Ka}^{2}} \\
& \sim\left(\frac{l}{\delta_{\mathrm{th}}}\right)^{4 / 3} \mathrm{Ka}^{2 / 3},
\end{aligned}
$$

where $\mathrm{Da}=l S_{L} / u^{\prime} \delta_{\text {th }}$ is the Damköhler number and $\mathrm{Ka}=\left(u^{\prime} / S_{L}\right)^{3 / 2} /\left(l / \delta_{\text {th }}\right)^{1 / 2}$ is the Karlovitz number. Here, $\delta_{\text {th }}=\left(T_{\mathrm{ad}}-T_{0}\right) / \operatorname{Max}|\nabla \hat{T}|_{L}$ is the thermal flame thickness with $T_{\mathrm{ad}}, T_{0}$ and $\hat{T}$ being the adiabatic flame temperature, unburned gas temperature, and instantaneous dimensional temperature, respectively, while the subscript " $L$ " refers to the unstrained planar laminar flame quantities. Equation (9) indicates that a change of either Da or Ka will lead to a change in the turbulent Reynolds number $\operatorname{Re}_{t}$. Thus, the influence of $\mathrm{Re}_{t}$ on the statistics of $S_{d}$ is essentially induced by changes in $\mathrm{Da}$ and/or Ka.

\section{Numerical Implementation}

Three-dimensional compressible DNS runs for statistically planar turbulent premixed flames have been carried out using a DNS code called SENGA [28] in which the conservation equations of mass, momentum, energy, and species are solved in nondimensional form. The simulations have been carried out in a rectangular domain of size $36.6 \delta_{\text {th }} \times 24.1 \delta_{\text {th }} \times 24.1 \delta_{\text {th }}$. The simulation domain has been discretised using a Cartesian mesh of size $345 \times 230 \times$ 230 with uniform grid spacing in each direction. The mean direction of flame propagation is assumed to align with the $x_{1}$-direction and the transverse directions are assumed to be periodic. The boundaries in the direction of mean flame propagation are taken to be partially nonreflecting and the boundary conditions are specified using the NavierStokes characteristic boundary conditions (NSCBC) technique [29]. The spatial discretisation is carried out using a 10th order central difference scheme for the internal points and the order of discretisation drops gradually to a one-sided 2 nd order scheme at nonperiodic boundaries. The time advancement is carried out using a low storage 3rd order Runge-Kutta scheme [30]. The turbulent velocity fluctuations are initialised using an initially homogeneous isotropic field using a pseudospectral method proposed by Rogallo [31]. The combustion is initailised by a steady unstrained planar laminar premixed flame solution. For the present study, the thermophysical properties such as dynamic viscosity $\mu$, thermal conductivity $\lambda$, specific heats $C_{P}$ and $C_{V}$ and the density-weighted mass diffusivity $\rho D$ are taken to be constant and independent of temperature following several previous studies [11-18, 32-34]. For the present study, standard values are taken for Prandtl number $(\mathrm{Pr}=$ $0.7)$ and the ratio of specific heats $\left(\gamma=C_{P} / C_{V}=1.4\right)$. The flame Mach number is taken to be $\mathrm{Ma}=S_{L} / \sqrt{\gamma R T_{0}}=$ 0.014 . The Lewis number Le is taken to be equal to unity for all the cases considered here. As Da and Ka are often used to characterise the combustion situation on combustion regime diagram, the variation of $\mathrm{Re}_{t}$ in the present study is brought about by modifying $\mathrm{Da}$ and $\mathrm{Ka}$ independently of each other. In order to achieve this, the values of both $u^{\prime} / S_{L}$ and $l / \delta_{\text {th }}$ are modified as both $\mathrm{Da}=l S_{L} / \delta_{\text {th }} u^{\prime}$ and $\mathrm{Ka}=$ $\left(u^{\prime} / S_{L}\right)^{3 / 2}\left(l / \delta_{\text {th }}\right)^{-1 / 2}$ are dependent on the values of $u^{\prime} / S_{L}$ and $l / \delta_{\text {th }}$. The initial values of normalised rms turbulent velocity fluctuation $u^{\prime} / S_{L}$, integral length scale to flame thickness ratio $l / \delta_{\text {th }}$, heat release parameter $\tau=\left(T_{\text {ad }}-T_{0}\right) / T_{0}$, Damköhler number Da, Karlovitz number Ka and turbulent Reynolds number $\mathrm{Re}_{t}$ are listed in Table 1. From Table 1, it is evident that Ka remains greater than unity for all cases, which indicates that the combustion belongs to the thin reaction zones regime according to the regime diagram by Peters [1]. In cases $\mathrm{B}, \mathrm{C}$, and $\mathrm{D}$ the values of $u^{\prime} / S_{L}$ and $l / \delta_{\text {th }}$ were chosen in such a manner as to vary the turbulent Reynolds number $\mathrm{Re}_{t}$ by changing the Damköhler number Da while keeping the Karlovitz number Ka constant (see (9)). On the other hand, in cases $\mathrm{A}, \mathrm{C}$, and $\mathrm{E}, u^{\prime} / S_{L}$ and $l / \delta_{\text {th }}$ were chosen to vary $\mathrm{Re}_{t}$ by changing $\mathrm{Ka}$, while $\mathrm{Da}$ was kept constant. The range of turbulent Reynolds number values considered in this study remains modest although several previous studies [3-22] with comparable values of $\mathrm{Re}_{t}$ have made valuable contributions to the fundamental understanding of displacement speed in turbulent premixed flames. It is also worth noting that the range of $\mathrm{Re}_{t}$ considered here is comparable to that of previous laboratory-scale experiments (e.g., Kobayashi et al. [35]). Despite the limited range, a number of important $\mathrm{Re}_{t}$ effects on the displacement speed statistics have been identified in the present parametric study and are discussed in detail in Section 4 of this paper.

In all cases, the flame-turbulence interaction takes place under conditions of decaying turbulence, in which simulations should be carried out for a minimum time $t_{\text {sim }}=$ $\operatorname{Max}\left(t_{f}, t_{c}\right)$, where $t_{f}=l / u^{\prime}$ is the initial eddy turn over time and $t_{c}=\delta_{\mathrm{th}} / S_{L}$ is the chemical time scale. All the cases considered here were run for one chemical time scale $t_{c}$, which corresponds to a time equal to $2.0 t_{f}$ in case $\mathrm{D}$, $3.0 t_{f}$ in cases $\mathrm{A}, \mathrm{C}$, and $\mathrm{E}$, and $4.34 t_{f}$ for case B. The present simulation time is comparable to the simulation times used for previous DNS studies which focused on the analysis of displacement speed statistics [3-21, 23-27]. By the time statistics were extracted, the global turbulent kinetic energy and volume-averaged burning rate were no longer changing rapidly with time. The temporal variations of these quantities were presented in [36], and thus are not repeated here for the sake of conciseness. Analysis of the volume-averaged burning rate indicates that the effects of unsteadiness have become weak by the time when statistics were extracted. At this time, 


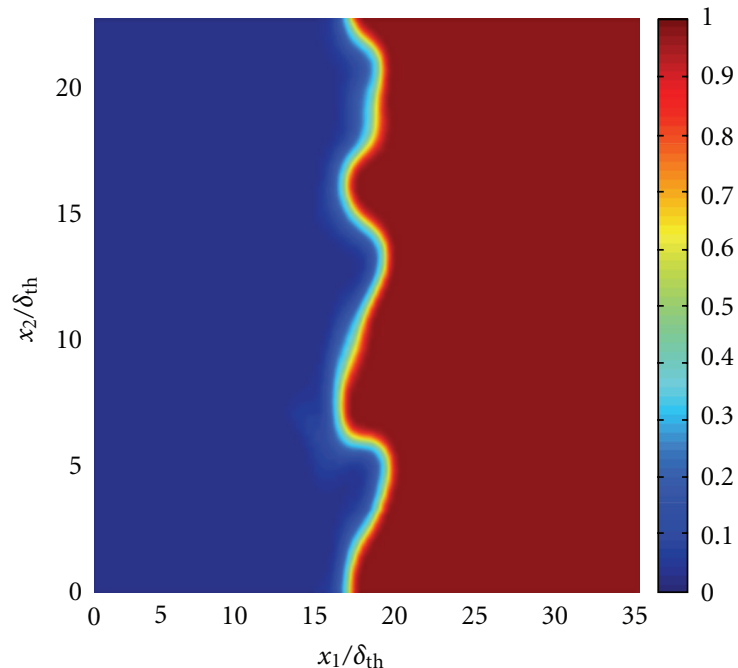

(a)

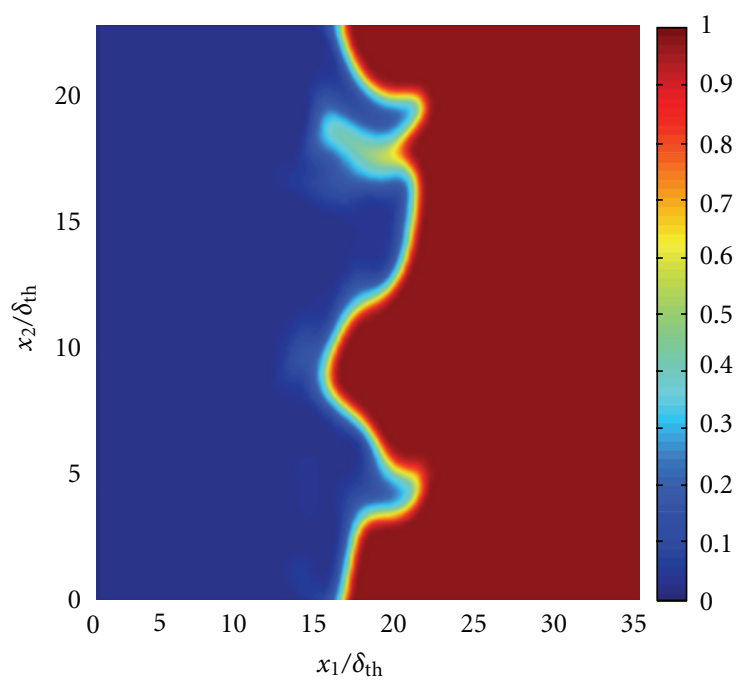

(c)

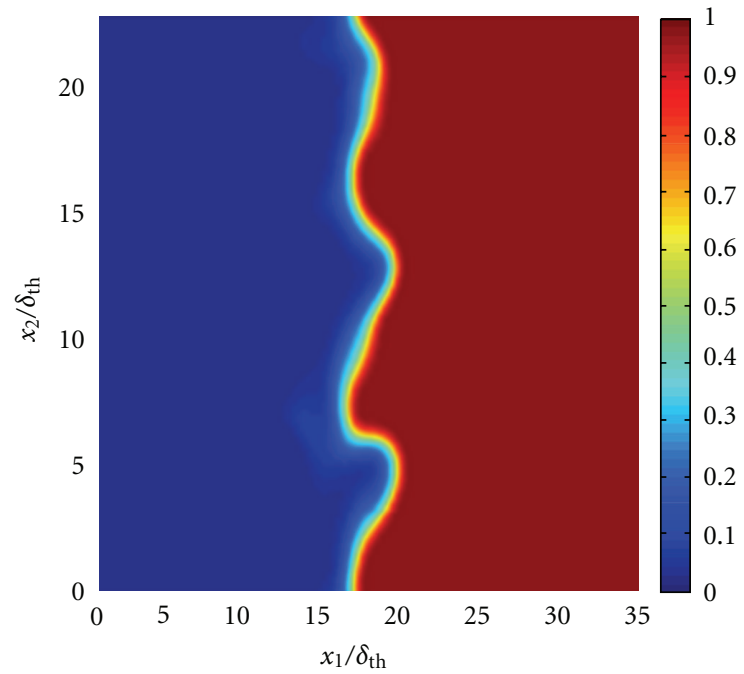

(b)

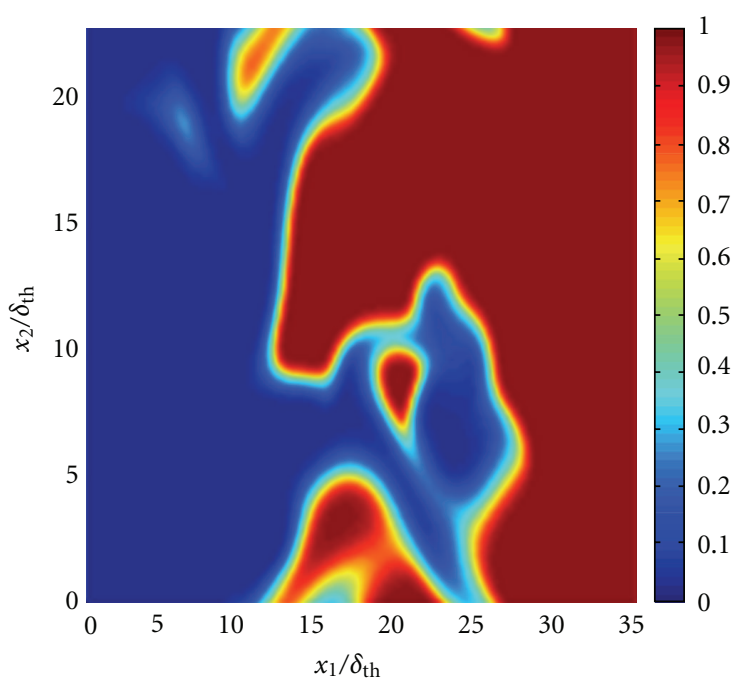

(d)

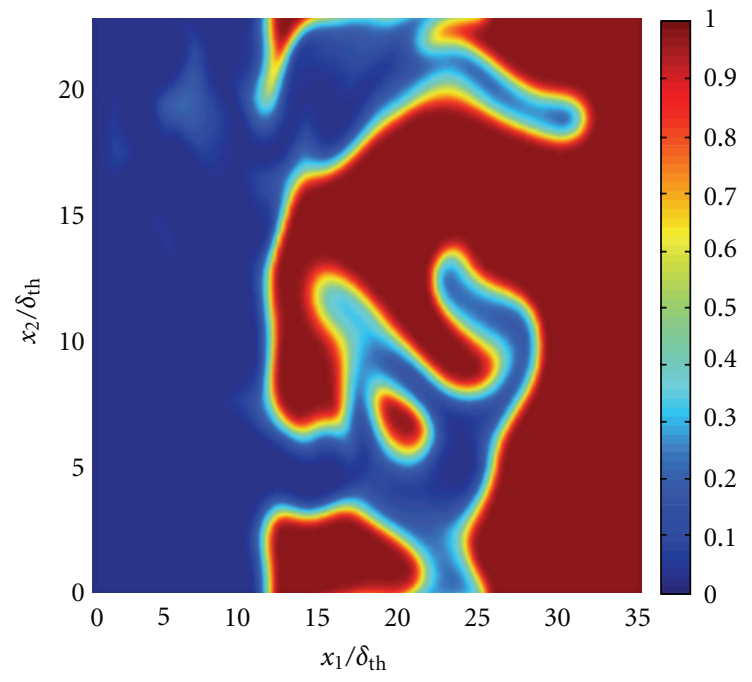

(e)

Figure 1: Contours of reaction progress variable $c$ in the $x_{1}-x_{2}$ midplane of the domain after $t=1.0 \delta_{\text {th }} / S_{L}$ for: (a) case A; (b) case B; (c) case C; (d) case D; (e) case E. 
TABLE 1: Simulation parameters and nondimensional numbers relevant to the DNS database.

\begin{tabular}{lcccccc}
\hline Case & $u^{\prime} / S_{L}$ & $l / \delta_{\text {th }}$ & $\tau$ & $\mathrm{Re}_{t}$ & $\mathrm{Da}$ & $\mathrm{Ka}$ \\
\hline $\mathrm{A}$ & 5.0 & 1.67 & 4.5 & 22 & 0.33 & 8.67 \\
$\mathrm{~B}$ & 6.25 & 1.44 & 4.5 & 23.5 & 0.23 & 13.0 \\
$\mathrm{C}$ & 7.5 & 2.5 & 4.5 & 49.0 & 0.33 & 13.0 \\
$\mathrm{D}$ & 9.0 & 4.31 & 4.5 & 100.0 & 0.48 & 13.0 \\
$\mathrm{E}$ & 11.25 & 3.75 & 4.5 & 110 & 0.33 & 19.5 \\
\hline
\end{tabular}

the global level of turbulent velocity fluctuation had decayed by $52.66 \%, 61.11 \%, 45 \%, 24 \%$, and $34 \%$ in comparison to the initial values for cases A to E, respectively. By contrast, the integral length scale had increased by factors of between 1.5 to 2.25 , ensuring that sufficient numbers of turbulent eddies were retained in each direction to obtain useful statistics. The values for $u^{\prime} / S_{L}, l / \delta_{\text {th }}$, and $\delta_{\text {th }} / \eta$ at the time when statistics were extracted have been presented in Table 2 of [36]. For all cases, the thermal flame thickness $\delta_{\text {th }}$ remains greater than the Kolmogorov length scale $\eta$ at that time, which indicates the combustion in all cases still belongs to the thin reaction zones regime. Moreover, the turbulent Reynolds number $\mathrm{Re}_{t}$ for all cases remains greater than unity (i.e., $\operatorname{Re}_{t}>1$ ) for the values of $u^{\prime} / S_{L}$ and $l / \delta_{\text {th }}$ at the time when statistics were extracted, which suggests that all the flames remain turbulent according to the regime diagram by Peters [1].

\section{Results and Discussion}

4.1. Flame-Turbulence Interaction. The contours of the reaction progress variable $c$ in the $x_{1}-x_{2}$ mid-plane of the domain at time $t_{\text {sim }}=1.0 \delta_{\text {th }} / S_{L}$ are shown in Figures 1(a)$1(\mathrm{e})$ for cases $\mathrm{A}$ to $\mathrm{E}$, respectively. It is evident that the extent of the wrinkling of the $c$ isosurfaces increases with increasing $u^{\prime}$, that is, in going from case A through to case E (see Table 1). Moreover, the contours of $c$ representing the preheat zone (i.e., $c<0.5)$ are much more distorted than those representing the reaction zone (i.e., $0.7<c<$ $0.9)$. This tendency is more prevalent for the case with the highest Karlovitz number (case E), since the scale separation between $\delta_{\text {th }}$ and $\eta$ increases with increasing $\mathrm{Ka}$, allowing more energetic eddies to enter into the preheat zone and causing greater distortion of the flame.

4.2. Probability Density Functions of Displacement Speed $S_{d}$. The probability density functions (pdfs) of normalised displacement speed $S_{d} / S_{L}$ on the $c=0.1,0.3,0.5,0.7$, and 0.9 isosurfaces are shown in Figures 2(a)-2(e), respectively. It is evident in all cases that there is a small but finite probability of finding negative displacement speed although the mean value of $S_{d} / S_{L}$ remains strongly positive throughout the flame brush. Negative values of displacement speed suggest that the progress variable isosurface in question is moving locally in a direction opposite to that of mean flame propagation. This situation arises when the negative contribution of the molecular diffusion rate dominates over the positive semidefinite chemical reaction rate, as discussed in detail by Gran et al. [4]. Moreover, Figure 2(e) indicates that the probability of finding negative values of $S_{d} / S_{L}$ is greatest for the highest Karlovitz number (case E). This behaviour can be explained in terms of the scaling analysis of Peters [1] for unity Lewis number flames, which suggested that the different components of displacement speed scale as

$$
\frac{S_{r}+S_{n}}{v_{\eta}} \sim \frac{S_{L}}{v_{\eta}} \sim O\left(\frac{1}{\sqrt{\mathrm{Ka}}}\right), \quad \frac{S_{t}}{v_{\eta}} \sim-\frac{2 D \kappa_{m}}{v_{\eta}} \sim O(1),
$$

where $v_{\eta}$ is the Kolmogorov velocity scale. Here, in accordance with Peters [1], $\left(S_{r}+S_{n}\right)$ is taken to scale with $S_{L}$ and the curvature $\kappa_{m}$ is taken to scale with the Kolmogorov length scale $1 / \eta$. These scalings of $\left(S_{r}+S_{n}\right)$ and $\kappa_{m}$ were confirmed by DNS data presented in [21]. Equation (10) clearly suggests that in the thin reaction zones regime (i.e., Ka $>1)$ the effects of $\left(S_{r}+S_{n}\right)$ are likely to weaken progressively in comparison to the contribution of $S_{t}$ with increasing Karlovitz number. This suggests that for large values of Karlovitz number the negative contribution of $S_{t}$ more readily overcomes the predominantly positive contribution of $\left(S_{r}+S_{n}\right)$ to yield a negative value of $S_{d}$. This eventually gives rise to an increased probability of finding negative values of $S_{d}$ for flames with increasing value of Karlovitz number, as suggested by Figure 2. In order to demonstrate that the observed negative values of $S_{d}$ originates principally due to $S_{t}$, the pdfs of $\left(S_{r}+S_{n}\right) / S_{L}$ are shown in Figure 3, respectively, for all cases. Comparing Figures 2 and 3 reveals that the probability of finding negative values of $\left(S_{r}+S_{n}\right)$ is significantly smaller than that of finding negative values of $S_{d}$.

Comparing Figures 2(a)-2(e) and Figures 3(a)-3(e), it can be seen that the most probable values of $S_{d} / S_{L}$ and $\left(S_{r}+S_{n}\right) / S_{L}$ remain of the order of $\rho_{0} / \rho$ and that the widths of the displacement speed pdf for a given $c$ isosurface increases with increasing $u^{\prime}$ (i.e., going from case $\mathrm{A}$ to case $\mathrm{E}$ ). In order to explain this behavior, it is useful to examine the pdfs of the normalised tangential component of displacement speed $S_{t} / S_{L}$ which are depicted in Figure 4 and show that these pdfs remain almost symmetrical with a peak close to a value of zero. This behaviour is consistent with the statistically planar nature of these flames. However, the greater extent of flame wrinkling observed in Figure 1 for larger values of $u^{\prime}$ results in a greater spread of flame curvature, which in turn increases the width of the $S_{t} / S_{L}$ pdfs according to (5). The negligible mean value of $S_{t}$ essentially leads to the mean values of $S_{d}$ and $\left(S_{r}+S_{n}\right)$ becoming almost equal to each other, but the statistical variation of $S_{d}$ induced by $S_{t}$ leads to broader pdfs of $S_{d}$ than those of $\left(S_{r}+S_{n}\right)$. These results clearly 


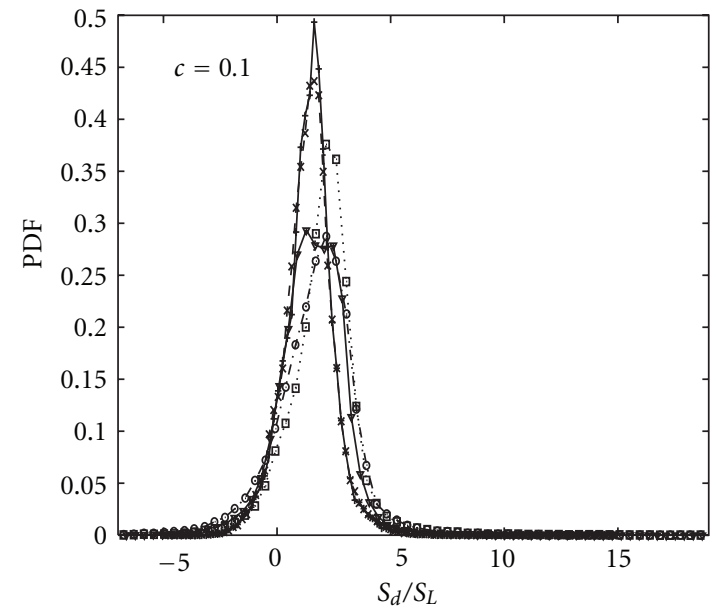

(a)

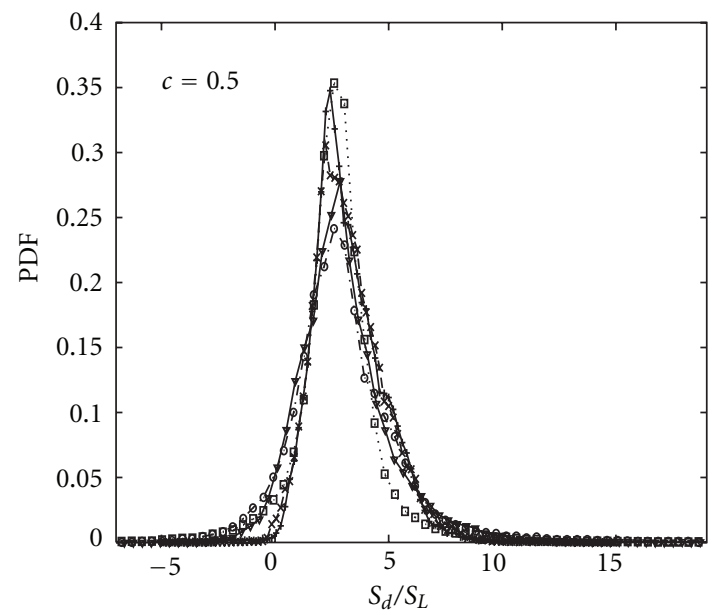

(c)

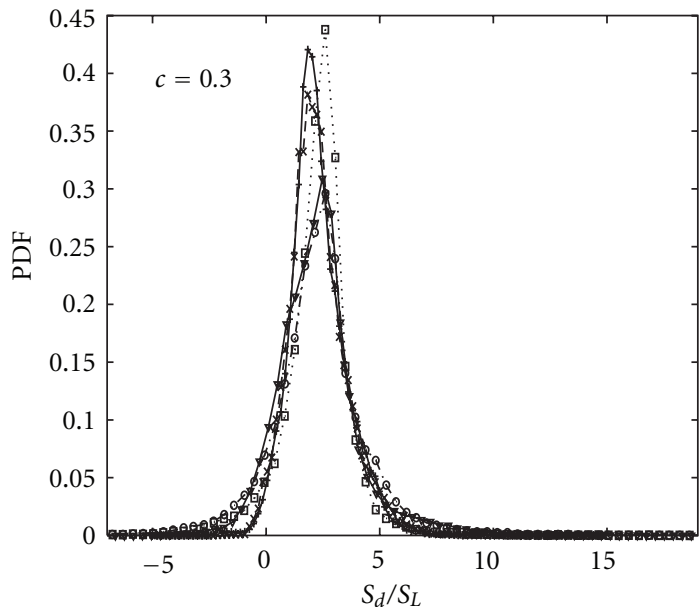

(b)

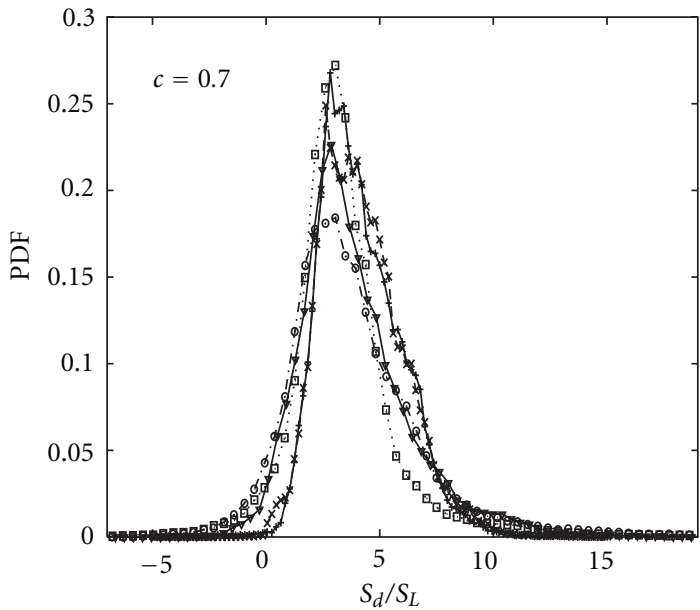

(d)

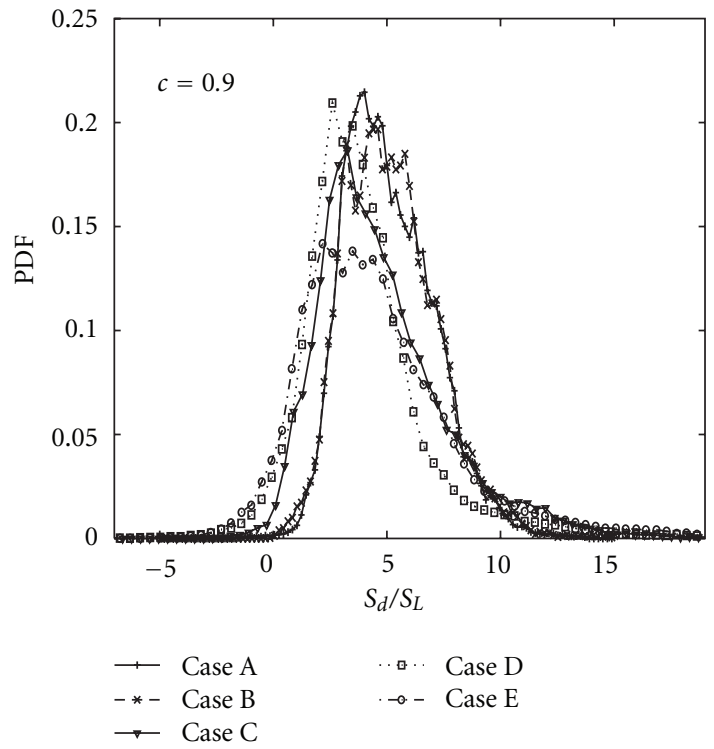

(e)

Figure 2: Pdfs of normalised displacement speed $S_{d} / S_{L}$ on the $c=$ (a) 0.1 , (b) 0.3 , (c) 0.5 , (d) 0.7 , and (e) 0.9 isosurfaces. 


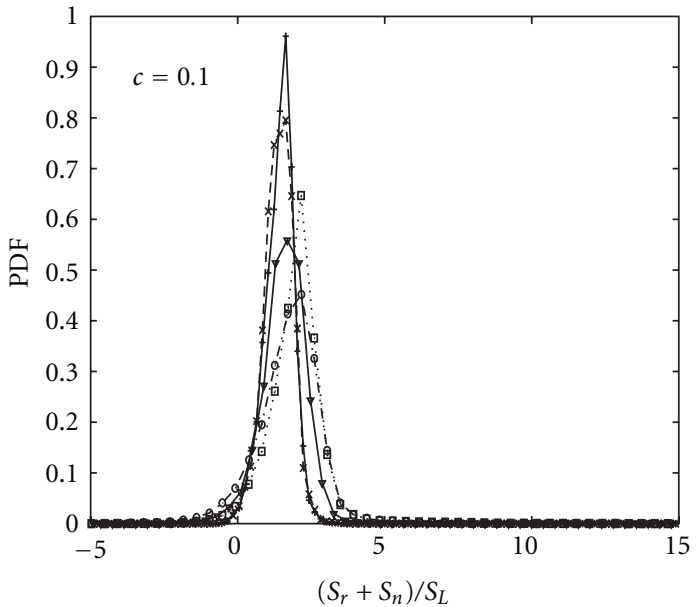

(a)

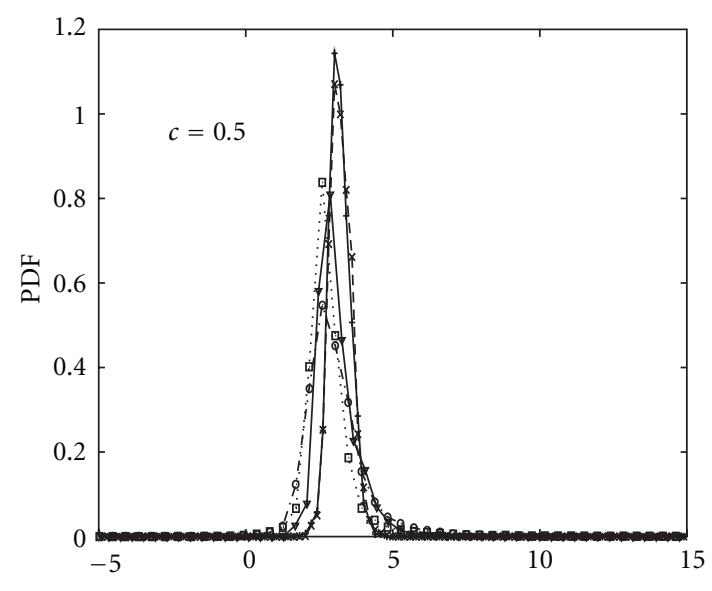

$\left(S_{r}+S_{n}\right) / S_{L}$

(c)

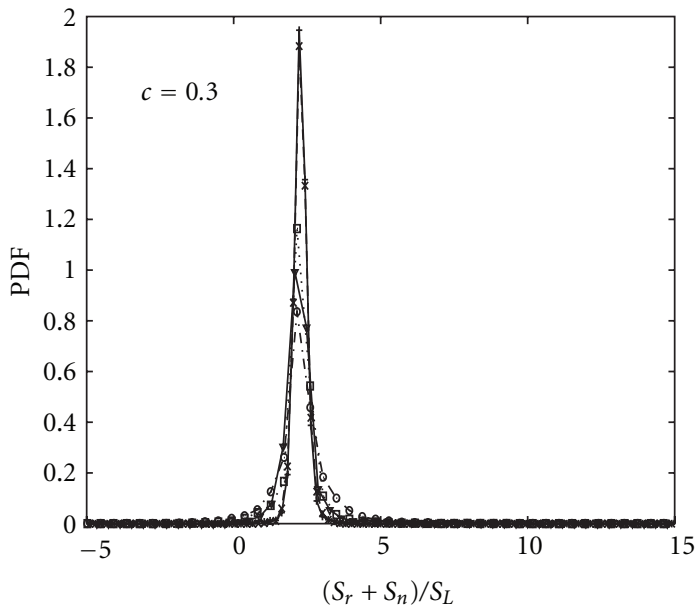

(b)

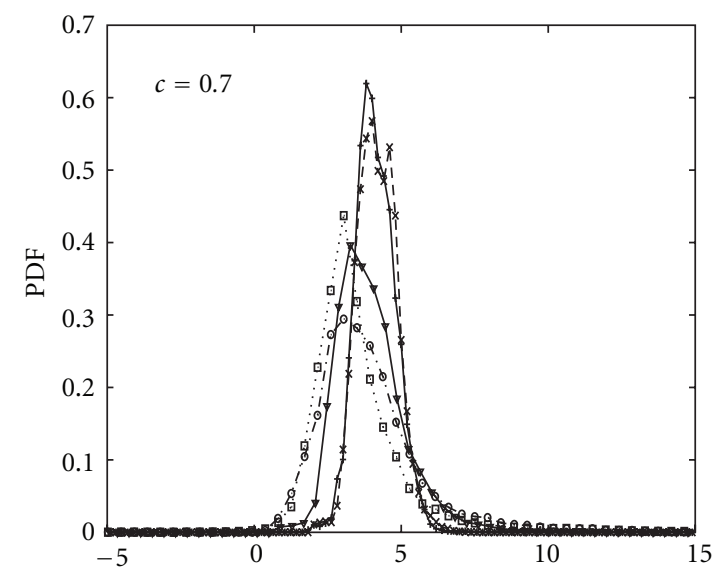

$\left(S_{r}+S_{n}\right) / S_{L}$

(d)

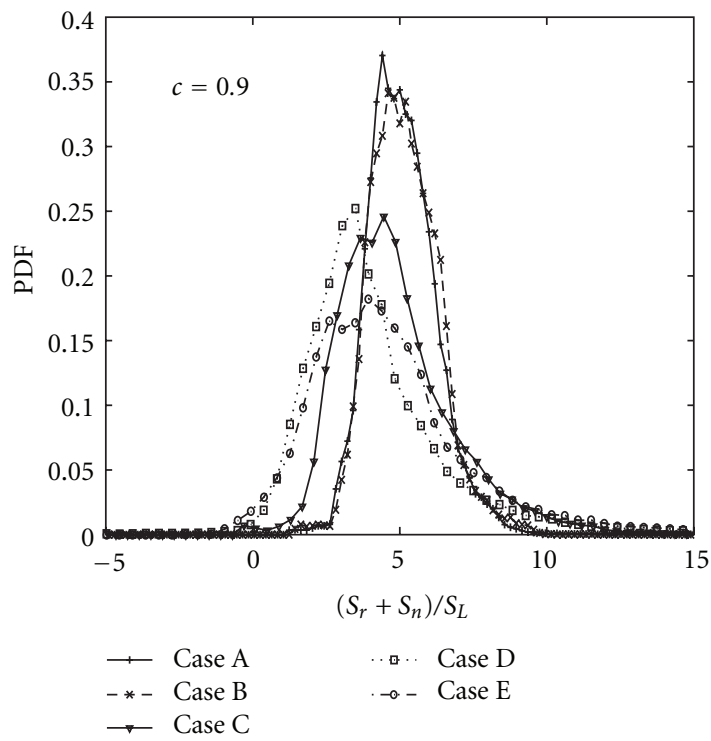

(e)

Figure 3: Pdfs of normalised combined reaction and normal diffusion component of displacement speed $\left(S_{r}+S_{n}\right) / S_{L}$ on the $c=$ (a) 0.1 , (b) 0.3 , (c) 0.5 , (d) 0.7 , and (e) 0.9 isosurfaces. 


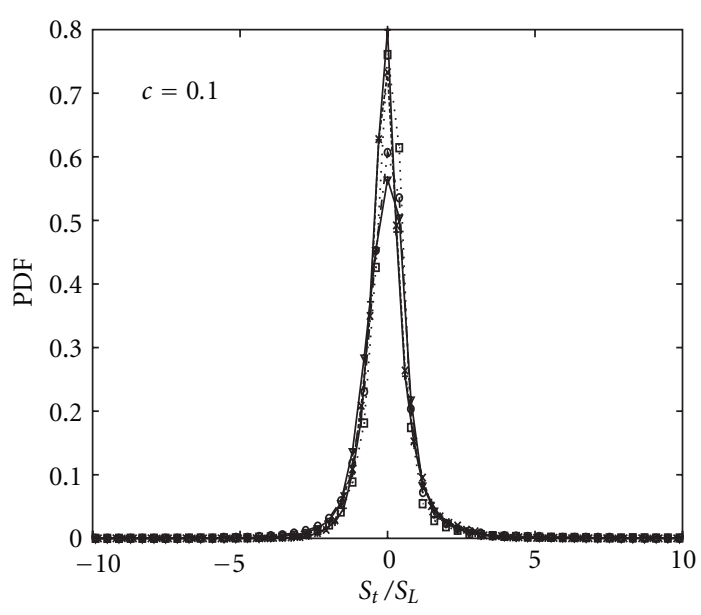

(a)

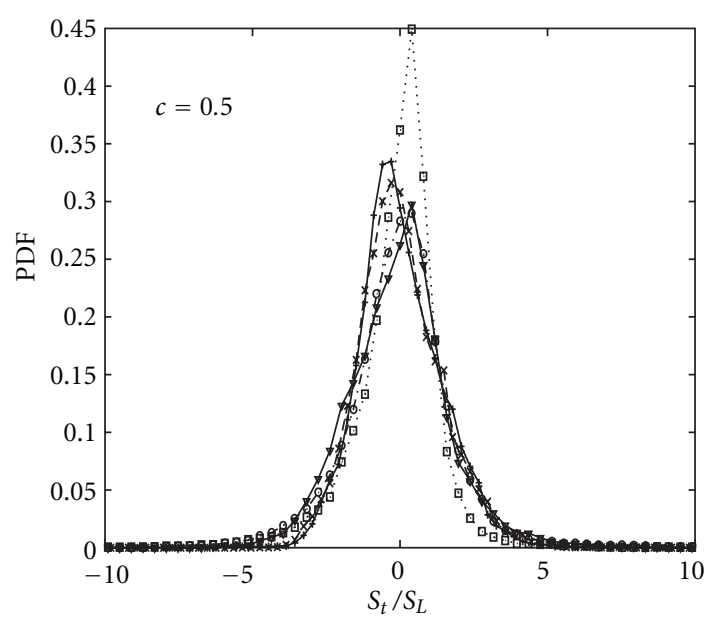

(c)

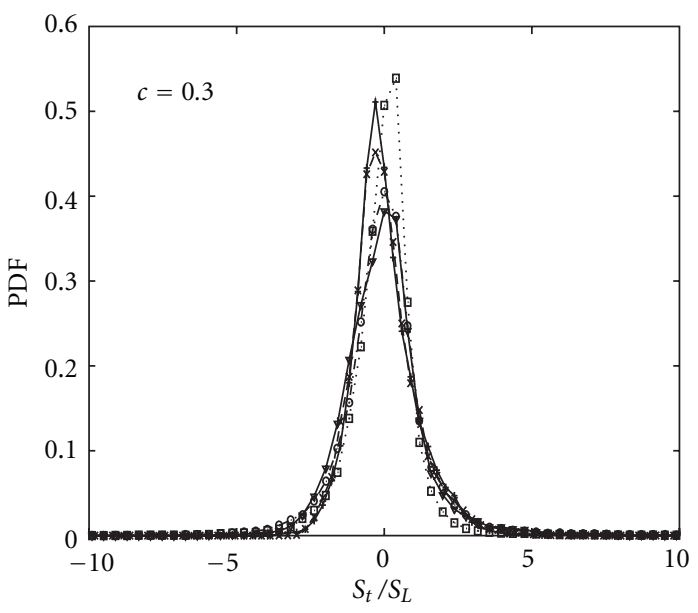

(b)

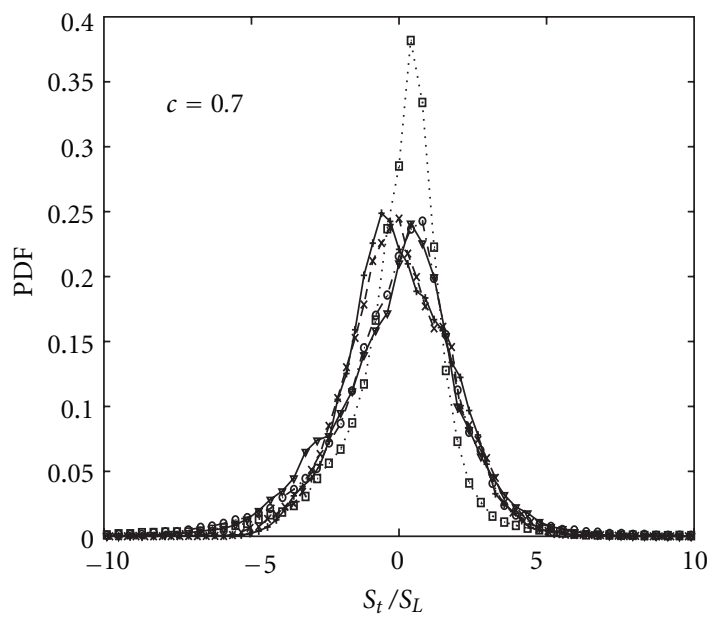

(d)

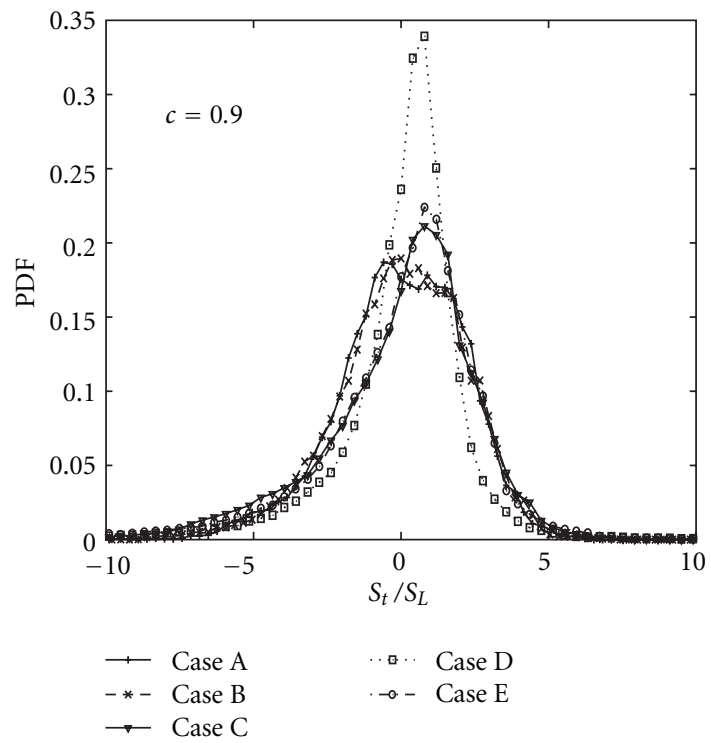

(e)

Figure 4: Pdfs of normalised tangential diffusion component of displacement speed $S_{t} / S_{L}$ on the $c=$ (a) 0.1 , (b) 0.3 , (c) 0.5 , (d) 0.7 , and (e) 0.9 isosurfaces. 
suggest that the distributions of $S_{d}$ and its components $S_{r}, S_{n}$ and $S_{t}$ generally broaden with $u^{\prime} / S_{L}$, which essentially leads to broadening of the distributions with increasing $\mathrm{Re}_{t} \sim\left(u^{\prime} / S_{L}\right)^{2} \mathrm{Da} \sim\left(u^{\prime} / S_{L}\right)^{4} / \mathrm{Ka}^{2} \sim\left(l / \delta_{\mathrm{th}}\right)^{4 / 3} \mathrm{Ka}^{2 / 3}$ when either $\mathrm{Da}$ or Ka is held constant.

4.3. Strain Rate and Curvature Dependences of $|\nabla c|$. For low Mach number unity Lewis number flames, both density $\rho$ and nondimensional temperature $T$ remain uniform on a given isosurface of $c$ and thus the statistical behaviours of $S_{r}$ and $S_{n}$ (see (5)) are mainly affected by the dependence of the SDF $|\nabla c|$ on strain rate and curvature. The values of $|\nabla c| \times \delta_{\text {th }}$ conditional on normalised tangential strain rate $a_{T} \times \delta_{\mathrm{th}} / S_{L}$ are shown in Figure 5(a) for the isosurface at $c=0.8$ (close to the location of maximum reaction rate), which suggests clearly that $|\nabla c|$ and $a_{T}$ are positively correlated for all cases. The correlation coefficients between $|\nabla c|$ and $a_{T}$ are shown in Figure 5(b) for values of $c$ between 0.1 and 0.9 , and these indicate that the positive correlation is maintained throughout the flame brush. This correlation can be explained by noting that the dilatation rate $\nabla \cdot \vec{u}$ can be expressed as

$$
\nabla \cdot \vec{u}=a_{T}+a_{n} .
$$

For unity Lewis number flames, the dilatation rate can be scaled as $\nabla \cdot \vec{u} \sim \tau S_{L} / \delta_{\text {th }}[21]$, whereas $a_{T}$ can be scaled as $a_{T} \sim u^{\prime} / l[37]$, which suggests that

$$
\frac{\nabla \cdot \vec{u}}{a_{T}} \sim \tau \frac{S_{L} l}{\delta_{\mathrm{th}} u^{\prime}} \sim \tau \mathrm{Da} .
$$

Equation (11b) essentially shows that for low values of the Damköhler number, $a_{T}$ remains much greater than $\nabla$. $\vec{u}$. This essentially suggests that an increase in tangential strain rate is associated with a decrease in the normal strain rate, since the normal strain rate scales as $a_{n} \sim-a_{T}$ when dilatation rate effects are much weaker than those of turbulent straining. Under decreasing normal straining, the isoscalar lines are brought closer to each other and hence give rise to an increase in $|\nabla c|$. The decreasing trend of normal strain rate with increasing tangential strain rate is reflected in the positive correlation between $|\nabla c|$ and $a_{T}$ throughout the flame brush. It can be seen from Figure 5 that the correlation between $|\nabla c|$ and $a_{T}$ does not show any monotonic trend with either Karlovitz number (Damköhler number) or turbulent Reynolds number variations when Damköhler number (Karlovitz number) is held constant.

The variation of $|\nabla c| \times \delta_{\text {th }}$ conditional on normalised curvature $\kappa_{m} \times \delta_{\text {th }}$ is shown in Figure 6(a) for the isosurface at $c=0.8$. Both positive and negative correlation trends can be observed, which is consistent with several previous studies [14-22, 26, 27]. As a result of these opposing trends, the net correlation between $|\nabla c| \times \delta_{\text {th }}$ and $\kappa_{m} \times \delta_{\text {th }}$ turns out be weak throughout the flame brush, as evident from the correlation coefficients between $|\nabla c|$ and $\kappa_{m}$ presented for different values of $c$ across the flame brush in Figure 6(b). Both the positively and negatively correlating branches can be explained using (11a). In turbulent premixed flames the dilatation rate $\nabla \cdot \vec{u}$ is negatively correlated with curvature $\kappa_{m}$, because of focusing (defocusing) of heat in the negatively (positively) curved regions of the flame surface $[18,21]$. The tangential strain rate $a_{T}$ is also found to be negatively correlated with curvature $[18,21]$. The correlation coefficients for the $\nabla \cdot \vec{u}-\kappa_{m}$ and $a_{T}-\kappa_{m}$ correlations for different $c$ values across the flame brush are shown in Figures $7(\mathrm{a})$ and $7(\mathrm{~b})$, respectively. Comparing the correlation coefficients of the $\nabla \cdot \vec{u}-\kappa_{m}$ and $a_{T}-\kappa_{m}$ correlations for different cases reveals that the strength of both of these negative correlations decreases with increasing $u^{\prime} / S_{L}$ and $\mathrm{Re}_{t} \sim\left(l / \delta_{\mathrm{th}}\right)^{4 / 3} \mathrm{Ka}^{2 / 3}$ when either Da or Ka is held constant.

The combination of the positive correlation between $|\nabla c|$ and $a_{T}$ and the negative correlation between $a_{T}$ and $\kappa_{m}$ leads to small values of $|\nabla c|$ in regions of high positive curvature. This gives rise to the negative correlation trend between $|\nabla c|$ and $\kappa_{m}$, as observed in Figure 6. However, the dilatation rate $\nabla \cdot \vec{u}$ may locally attain large values in the highly negatively curved regions of the flame due to strong focussing of heat. Hence, $\nabla \cdot \vec{u}$ may locally exceed $a_{T}$ and induce an increase in $a_{n}$ according to (11a). As isoscalar lines move apart from each other under increasing normal strain rate, the increase in $a_{n}$ with increasing negative curvature leads to a positive correlation between $|\nabla c|$ and $\kappa_{m}$ in negatively curved regions, as also observed in Figure 6.

4.4. Curvature Dependence of $S_{d}$. The mean normalised displacement speed $S_{d} / S_{L}$ conditional on normalised curvature $\kappa_{m} \times \delta_{\text {th }}$ is shown in Figure 8(a) for $c=0.8$, which indicates that $S_{d} / S_{L}$ is negatively correlated with curvature and that the correlation is nonlinear in nature. These observations are consistent with several previous DNS studies with both detailed and simplified chemistry [3-11, 13-18, 20-22]. The variation of the correlation coefficient between $S_{d}$ and $\kappa_{m}$ for different $c$ isosurfaces across the flame brush is shown in Figure 8(b), which reveals that strength of the negative correlation decreases with increasing $\operatorname{Re}_{t} \sim\left(u^{\prime} / S_{L}\right)^{2} \mathrm{Da} \sim$ $\left(u^{\prime} / S_{L}\right)^{4} / \mathrm{Ka}^{2} \sim\left(l / \delta_{\mathrm{th}}\right)^{4 / 3} \mathrm{Ka}^{2 / 3}$ when either $\mathrm{Da}$ (see cases $\mathrm{A}, \mathrm{C}$, and $\mathrm{E}$ ) or $\mathrm{Ka}$ (see cases B, C, and D) is held constant (see Table 1). The tangential diffusion component of displacement speed $S_{t}$ is deterministically negatively correlated with curvature with correlation coefficient equal to unity for unity Lewis number flames (see (5)). Thus, the nonlinearity of the correlation between $S_{d}$ and $\kappa_{m}$ originates due to the curvature dependence of $S_{r}$ and $S_{n}$.

Mean values of the reaction component $S_{r} / S_{L}$ conditional on normalised curvature $\kappa_{m} \times \delta_{\text {th }}$ are shown in Figure 9(a) for the isosurface at $c=0.8$. The plot shows both positive and negative correlation trends. For low Mach number unity Lewis number flames, the curvature dependence of $S_{r}$ is principally determined by the curvature dependence of $|\nabla c|$. The negative (positive) correlation between $|\nabla c|$ and $\kappa_{m}$ ultimately gives rise to the positive (negative) correlation between $S_{r}$ and $\kappa_{m}$ as observed in Figure 9. As a result of the weak net correlation between $|\nabla c|$ and $\kappa_{m}$, the net correlation between $S_{r}$ and $\kappa_{m}$ also turns out to be 


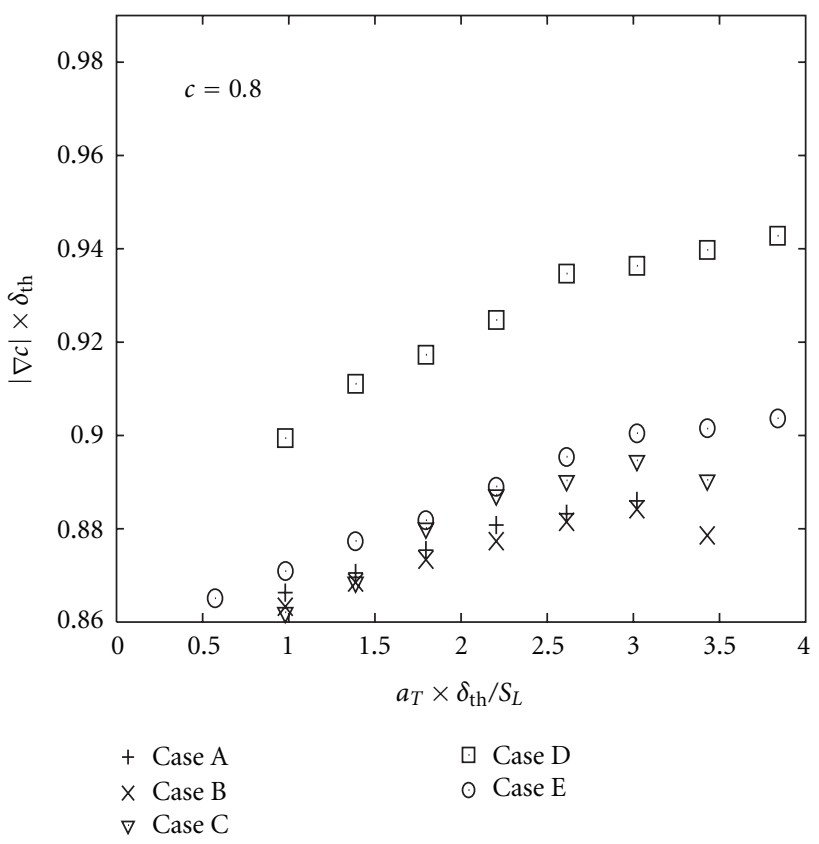

(a)

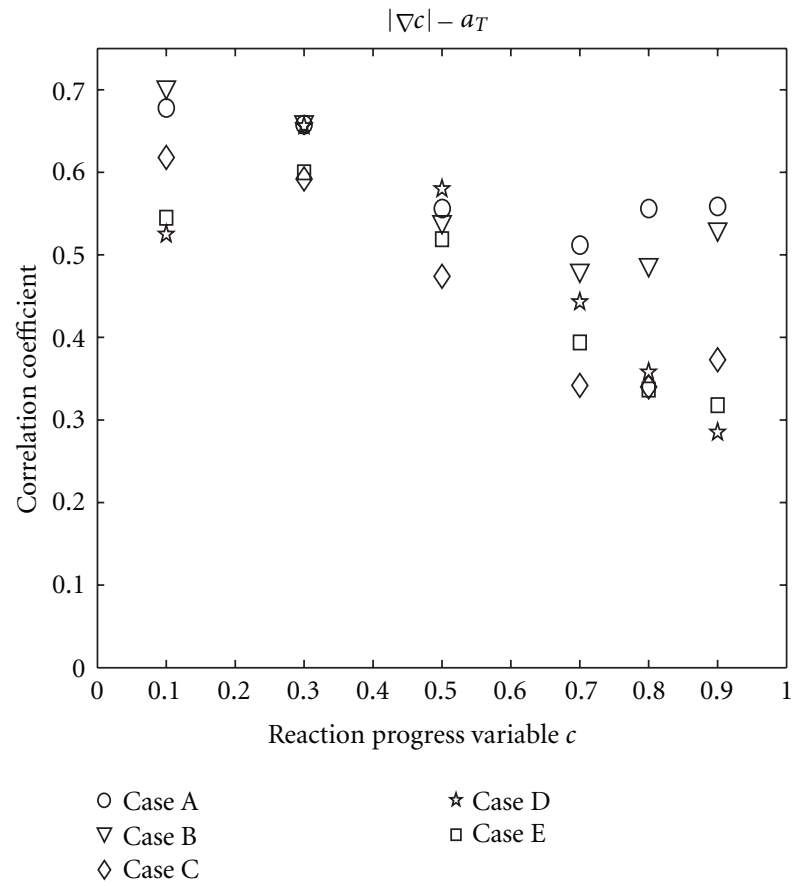

(b)

FIGURE 5: (a) Variation of mean values of $|\nabla c| \times \delta_{\text {th }}$ conditional on normalised tangential strain rate $a_{T} \times \delta_{\text {th }} / S_{L}$ for the isosurface at $c=0.8$. (b) Variation of the correlation coefficients between $|\nabla c|$ and $a_{T}$ on the isosurfaces at $c=0.1$ to 0.9 for all cases.

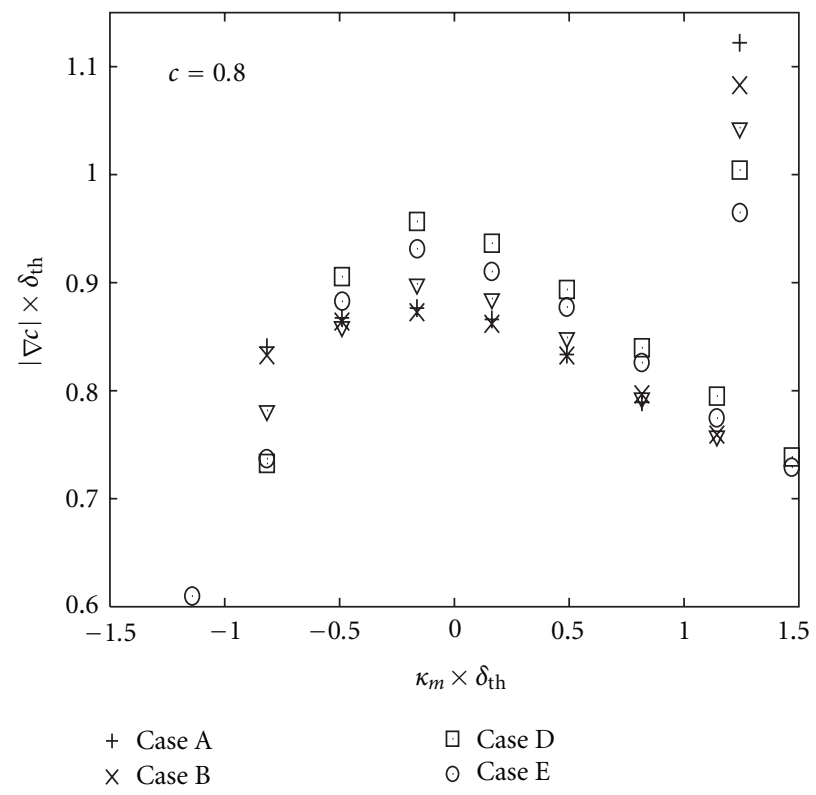

(a)

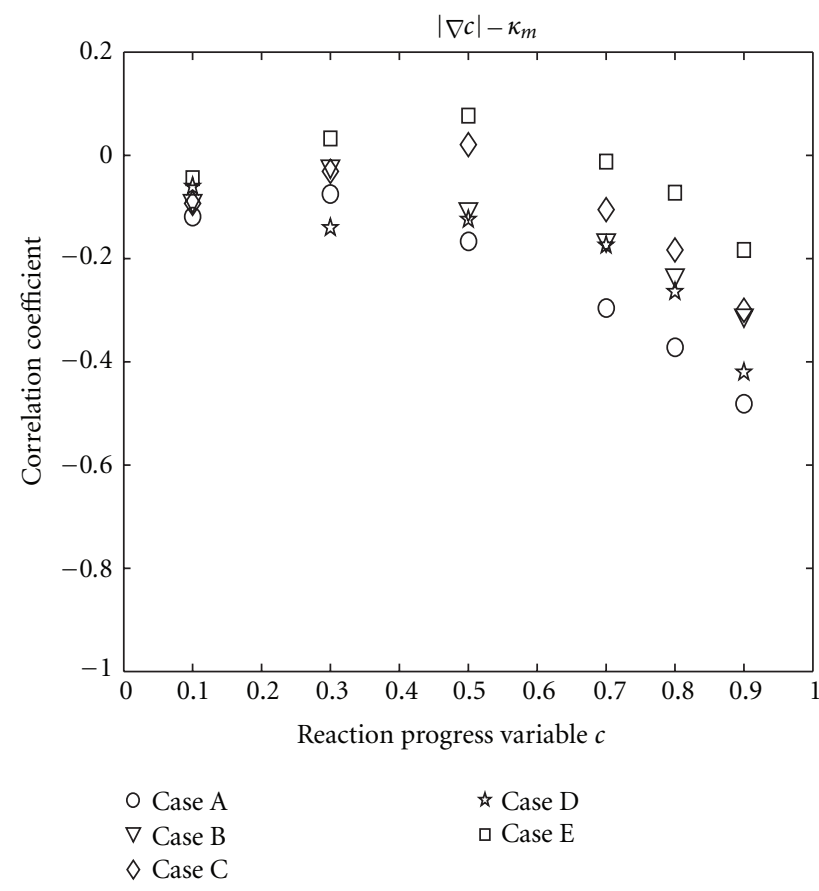

(b)

Figure 6: (a) Variation of mean values of $|\nabla c| \times \delta_{\text {th }}$ conditional on normalised curvature $\kappa_{m} \times \delta_{\text {th }}$ for the isosurface at $c=0.8$. (b) Variation of the correlation coefficients between $|\nabla c|$ and $\kappa_{m}$ on the isosurfaces at $c=0.1$ to 0.9 for all cases. 


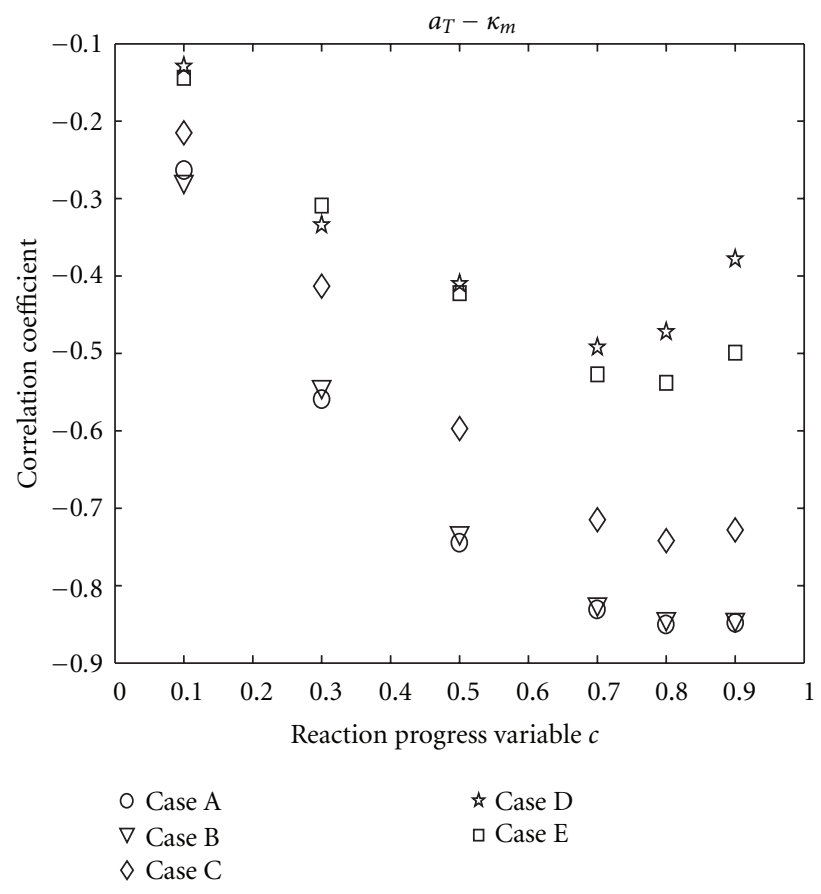

(a)

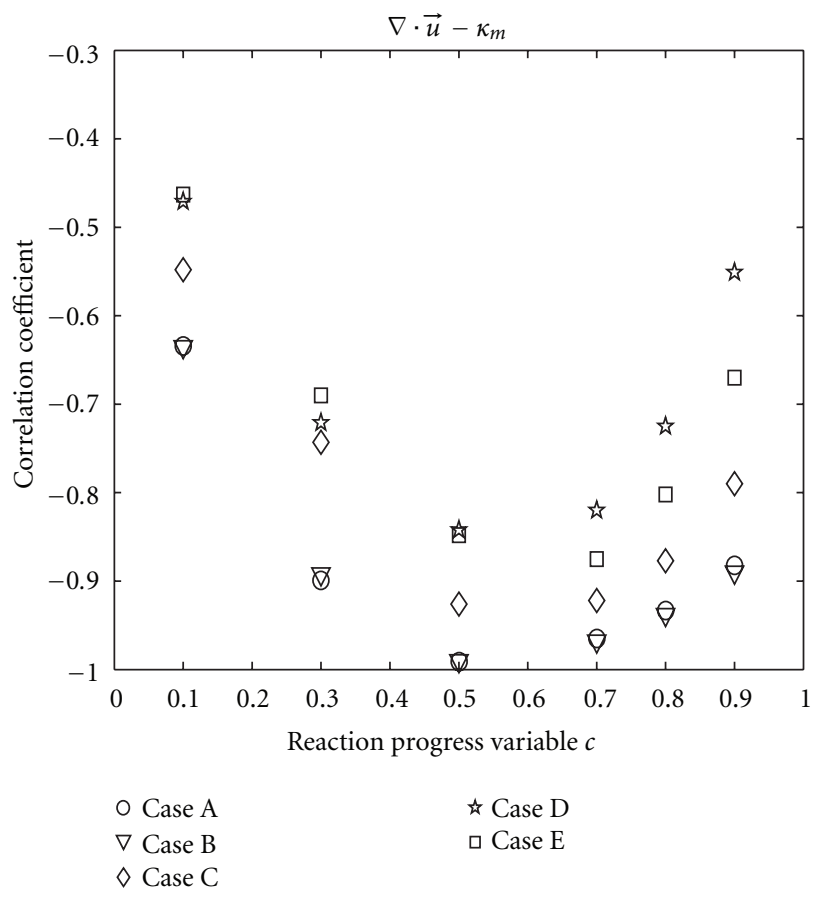

(b)

Figure 7: Variation of the correlation coefficients between (a) tangential strain rate $a_{T}$ and curvature $\kappa_{m}$ and between (b) dilatation rate $\nabla \cdot \vec{u}$ and curvature $\kappa_{m}$ on the isosurfaces at $c=0.1$ to 0.9 for all cases.

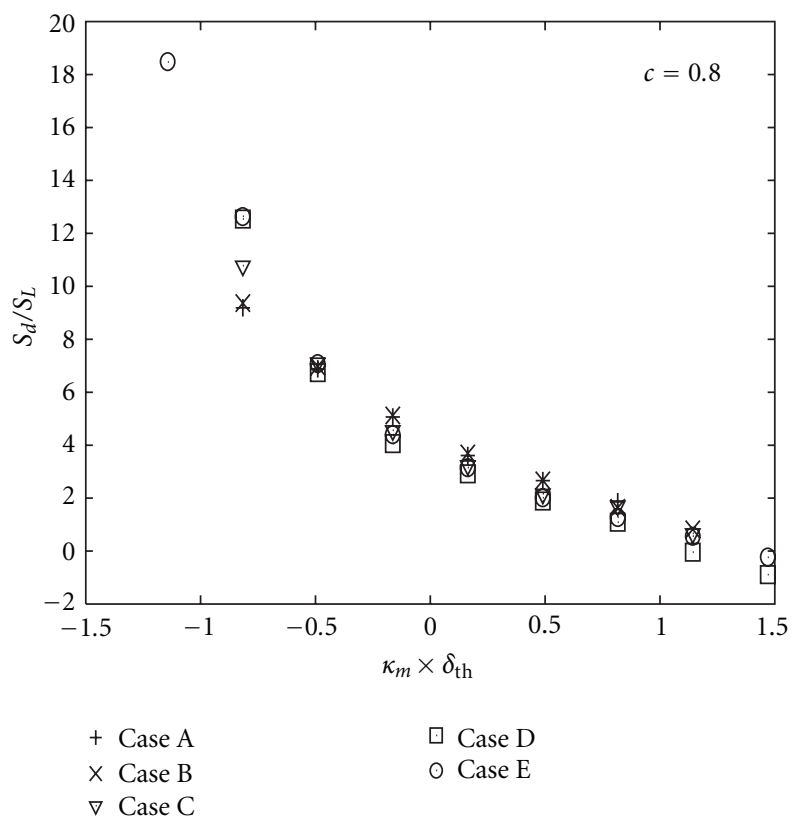

(a)
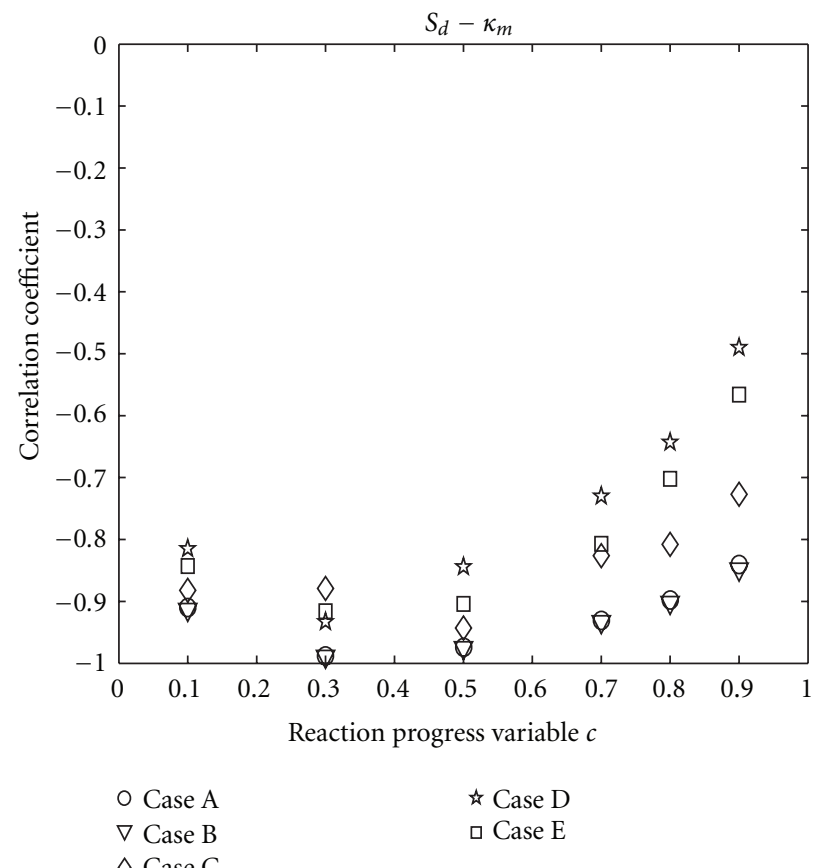

(b)

Figure 8: (a) Variation of mean values of $S_{d} / S_{L}$ conditional on normalised curvature $\kappa_{m} \times \delta_{\text {th }}$ for the isosurface at $c=0.8$. (b) Variation of the correlation coefficients between $S_{d}$ and $\kappa_{m}$ on the isosurfaces at $c=0.1$ to 0.9 for all cases. 


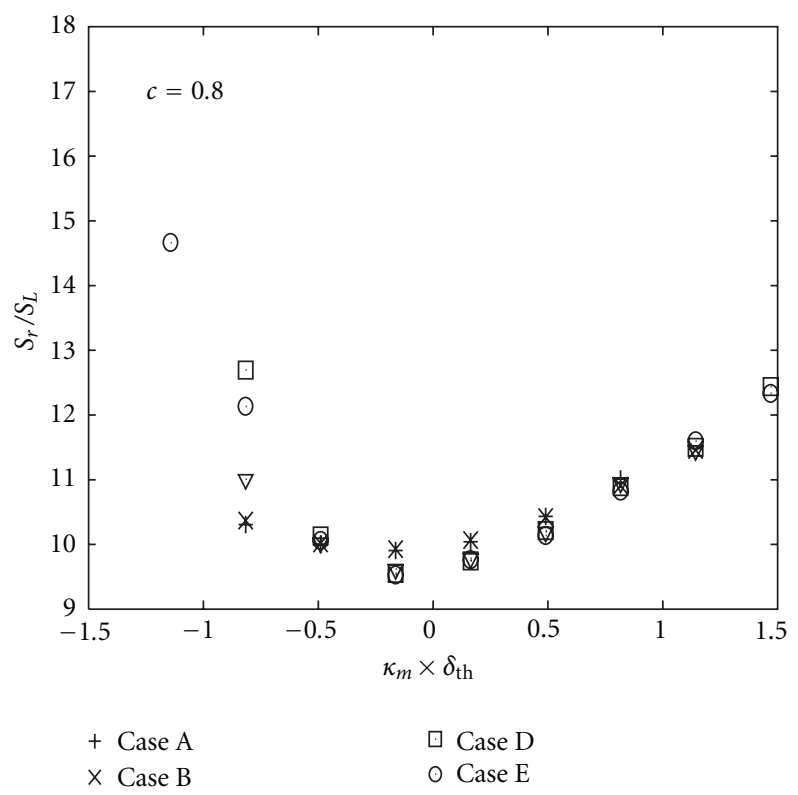

(a)

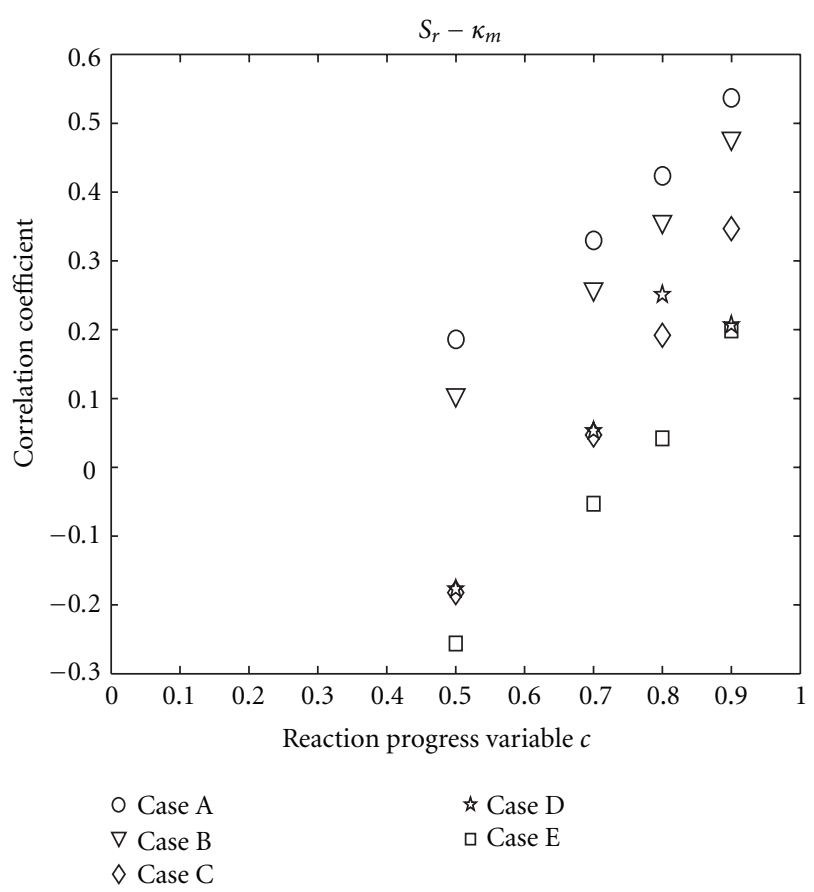

(b)

Figure 9: (a) Variation of mean values of $S_{r} / S_{L}$ conditional on normalised curvature $\kappa_{m} \times \delta_{\text {th }}$ for the isosurface at $c=0.8$. (b) Variation of the correlation coefficients between $S_{r}$ and $\kappa_{m}$ on the isosurfaces at $c=0.5$ to 0.9 for all cases.

weak, as indicated by the correlation coefficients plotted in Figure 9(b) Note that Figure 9(b) includes isosurfaces of $c$ from 0.5 to 0.9 only, since the reaction rate $\dot{w}$ is negligible in the preheat zone (see also (5)).

Corresponding mean values of the normal diffusion component $S_{n} / S_{L}$ conditional on normalised curvature $\kappa_{m} \times$ $\delta_{\text {th }}$ are shown in Figure 10(a) for $c=0.8$, while the correlation coefficients between $S_{n}$ and $\kappa_{m}$ are shown in Figure 10 (b) for values of $c$ from 0.1 to 0.9 . The magnitude of the normal molecular diffusion rate in the thin reaction zones regime can be scaled as $|\vec{N} \cdot \nabla(\rho D \vec{N} \cdot \nabla c)| \sim \rho D / \delta^{2}$ where $\delta$ is the characteristic flame thickness which in turn can be scaled as $|\nabla c| \sim 1 / \delta$. This suggests that $S_{n}$ scales as $D / \delta$ towards the reactant side of the flame brush and as $-D / \delta$ towards the product side. As a result of this, the positively (negatively) correlating branch of the $|\nabla c|-\kappa_{m}$ correlation gives rise to a positive (negative) correlation between $S_{n}$ and $\kappa_{m}$ towards the reactant side, where $S_{n}$ assumes predominantly positive values. By contrast, the positive (negative) correlation between $|\nabla c|$ and $\kappa_{m}$ results in a negative (positive) correlation between $S_{n}$ and $\kappa_{m}$ towards the product side where $S_{n}$ assumes predominantly negative values. Both positive and negative correlation trends can be discerned for all the $c$ isosurfaces shown in Figure 10(b). As a result of this, the net correlation between $S_{n}$ and $\kappa_{m}$ turns out to be weak throughout the flame brush. The positive and negative correlating trends with $\kappa_{m}$ observed for both $S_{r}$ and $S_{n}$ with $\kappa_{m}$ give rise to positive and negative correlating branches in the variation of $\left(S_{r}+S_{n}\right)$ conditional on $\kappa_{m} \times$ $\delta_{\text {th }}$ as shown in Figure 10(c) for $c=0.8$. This nonlinear correlation between $\left(S_{r}+S_{n}\right)$ and $\kappa_{m}$ ultimately induces a nonlinear curvature dependence of displacement speed $S_{d}$ (see Figure 8(a)), noting that the correlation coefficient arising from the deterministic relation between $S_{t}$ and $\kappa_{m}$ remains close to -1.0 throughout the flame brush for all cases. The correlation coefficients between $\left(S_{r}+S_{n}\right)$ and $\kappa_{m}$ are shown in Figure 10(d) for values of $c$ from 0.1 to 0.9, and demonstrate that the $\left(S_{r}+S_{n}\right)-\kappa_{m}$ correlation is much weaker than the $S_{t}-\kappa_{m}$ correlation in all cases. Hence, the net correlation between $S_{d}$ and $\kappa_{m}$ turns out to be negative throughout the flame brush for all the cases considered here (see Figure 8(a)).

4.5. Tangential Strain Rate Dependence of $S_{d}$. The mean values of normalised displacement speed $S_{d} / S_{L}$ conditional on normalised tangential strain rate $a_{T} \times \delta_{\mathrm{th}} / S_{L}$ are shown in Figure 11(a) for $c=0.8$ which suggests that $S_{d}$ and $a_{T}$ are positively correlated for all cases. The observed strain rate dependence of $S_{d}$ is found to be qualitatively consistent with previous DNS studies for flames with global Lewis number close to unity $[3,5,12,14,15,17-19,21,22]$. The variations of the correlation coefficients between $S_{d}$ and $a_{T}$ for different $c$ isosurfaces are shown in Figure 11(b), which reveals that the strength of the correlation between $S_{d}$ and $a_{T}$ decreases with increasing $u^{\prime} / S_{L}$ when either $\mathrm{Da}$ or Ka is held constant. This is consistent with the analytical treatment by Joulin [38] who indicated that the strain rate dependence of $S_{d}$ weakens with increasing frequency of straining. In order to explain the observed behaviour it is useful to look into the dependence of $S_{r}, S_{n}$, and $S_{t}$ on $a_{T}$. For low Mach number unity, Lewis 




(a)

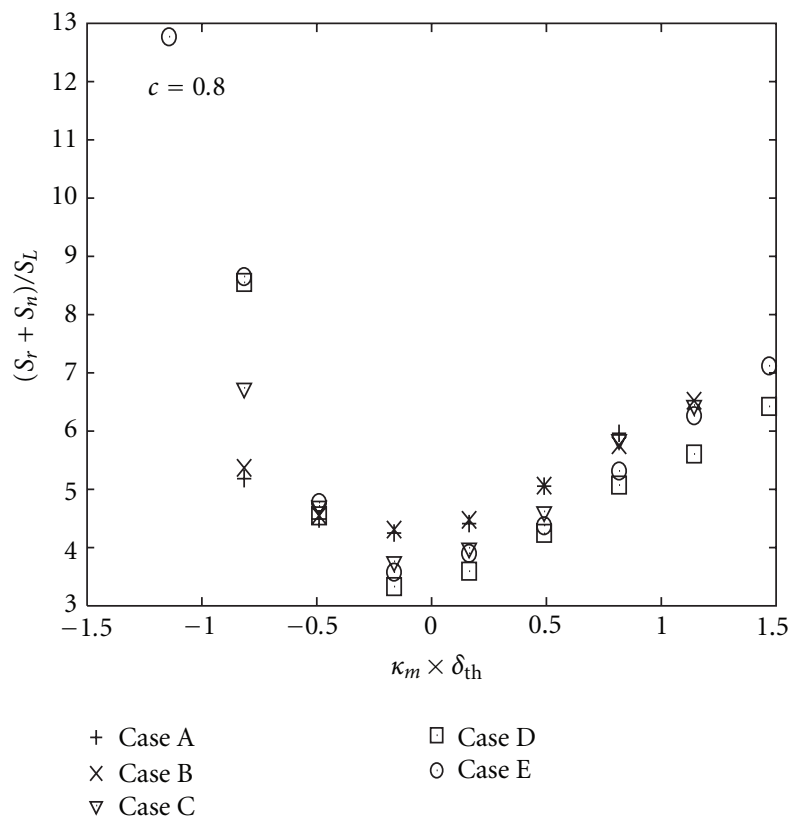

(c)

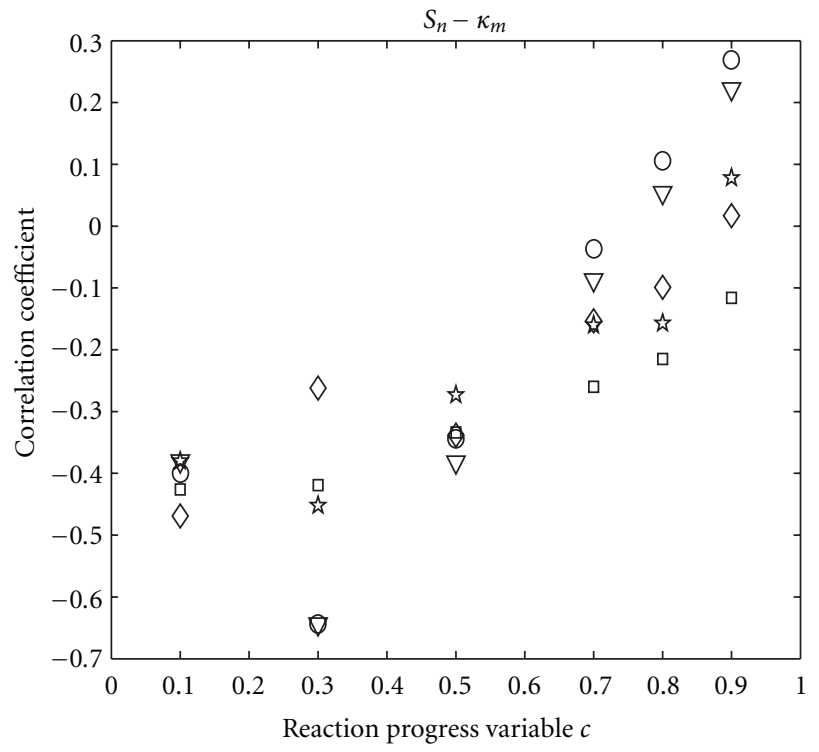

(b)

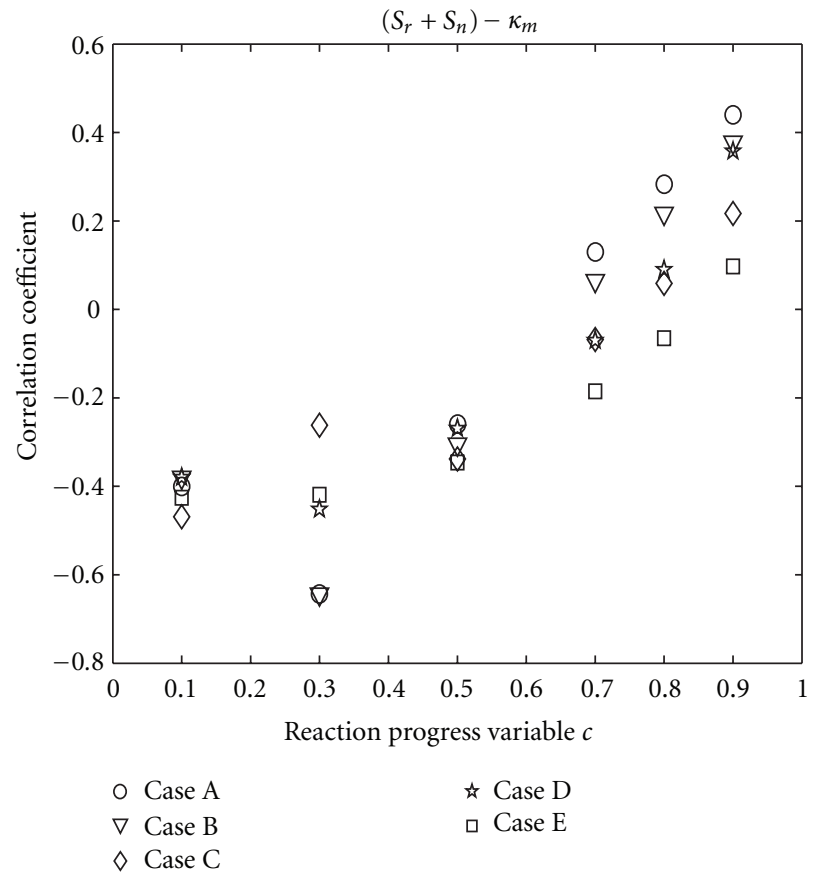

(d)

Figure 10: (a) Variation of mean values of $S_{n} / S_{L}$ conditional on normalised curvature $\kappa_{m} \times \delta_{\text {th }}$ for the isosurface at $c=0.8$. (b) Variation of the correlation coefficients between $S_{n}$ and $\kappa_{m}$ on the isosurfaces at $c=0.1$ to 0.9 for all cases. (c) Variation of mean values of $\left(S_{r}+S_{n}\right) / S_{L}$ conditional on normalised curvature $\kappa_{m} \times \delta_{\text {th }}$ for the isosurface at $c=0.8$. (d) Variation of the correlation coefficients between $\left(S_{r}+S_{n}\right)$ and $\kappa_{m}$ on the isosurfaces at $c=0.1$ to 0.9 for all cases.

number flames the strain rate dependence of $S_{r}$ is essentially determined by the strain rate dependence of $|\nabla c|$. It has been shown already that $|\nabla c|$ and $a_{T}$ are positively correlated (see Figure 5), which gives rise to a negative correlation between $S_{r}$ and $a_{T}$ according to (5). This is substantiated by the correlation coefficients between $S_{r}$ and $a_{T}$ as shown in Figure 11(c) for the isosurfaces from $c=0.5$ to 0.9. The correlation between $S_{r}$ and $a_{T}$ does not show any monotonic trend with either Karlovitz number (Damköhler number) or turbulent Reynolds number variations when the Damköhler number (Karlovitz number) is held constant, due to the lack of any such trend in the $|\nabla c|-a_{T}$ correlation.

It has been discussed already that $S_{n}$ scales as $S_{n} \sim D / \delta$ on the reactant side of the flame brush, and as $S_{n} \sim-D / \delta$ on the product side. Since there is a positive correlation between $|\nabla c| \sim 1 / \delta$ and $a_{T}$, this gives rise to a positive 


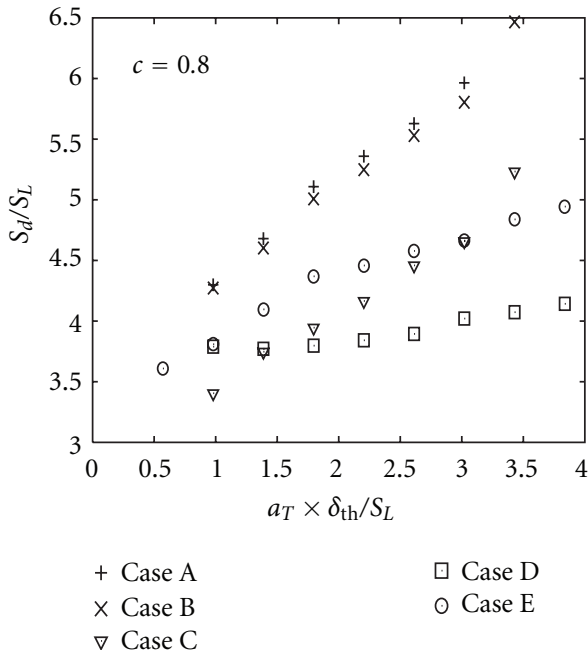

(a)

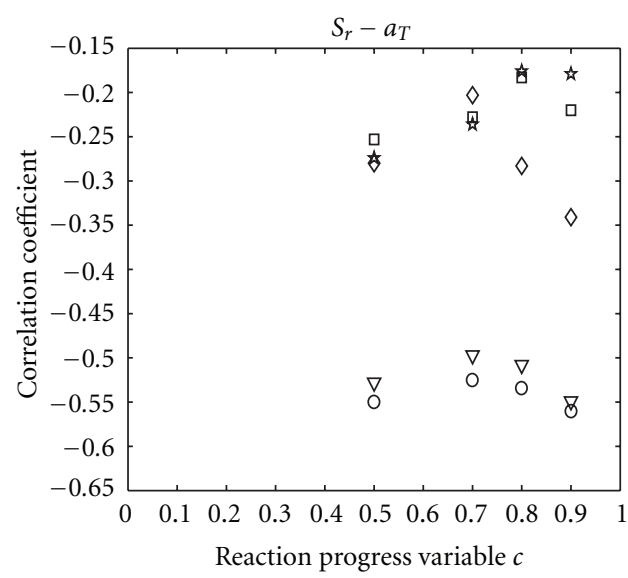

(c)

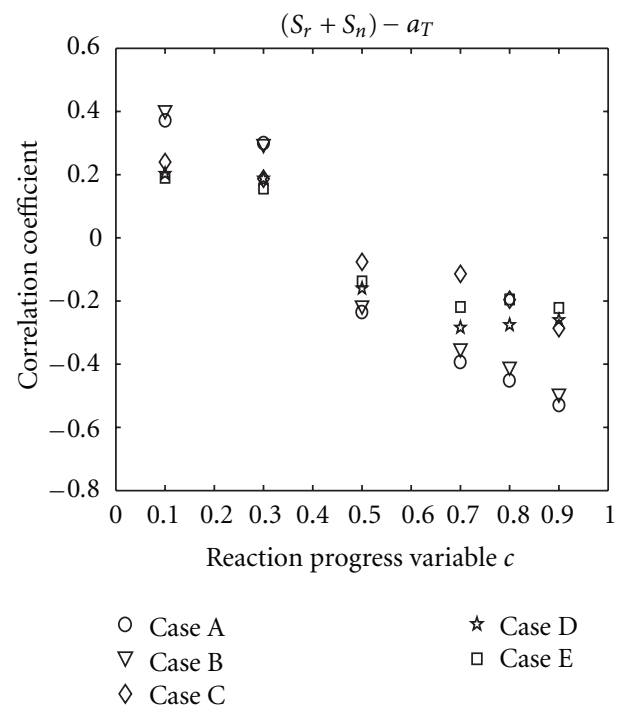

(e)
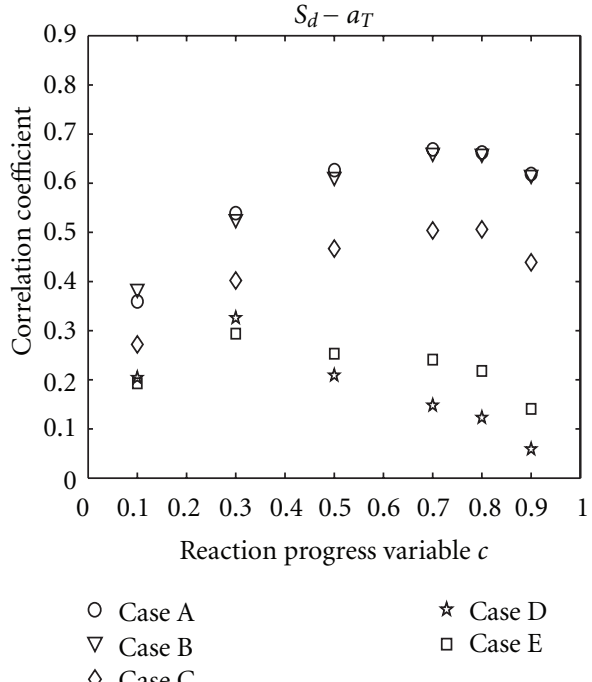

(b)

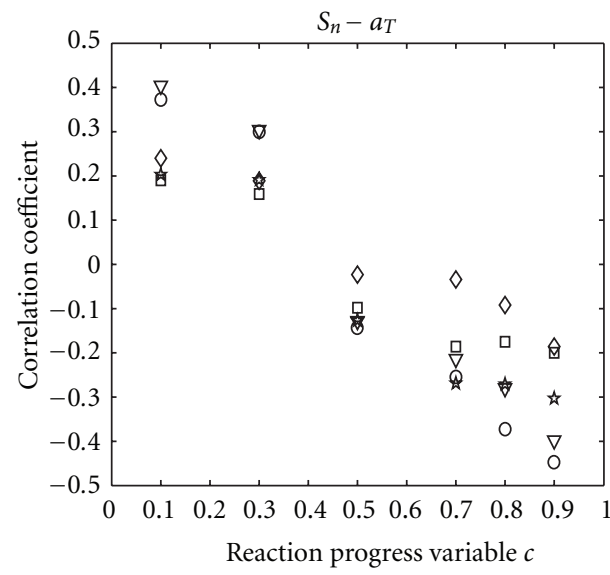

(d)

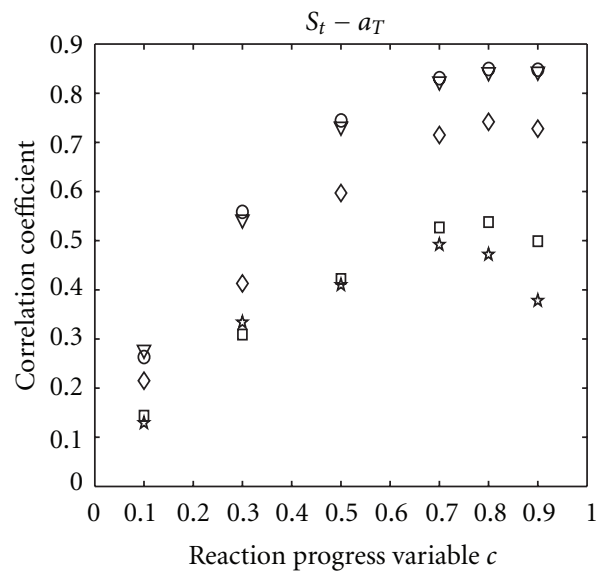

O Case A

$\nabla$ Case B

* Case D

๑ Case E

$\diamond$ Case C

Figure 11: (a) Variation of mean values of $S_{d} / S_{L}$ conditional on normalised tangential strain rate $a_{T} \times \delta_{\mathrm{th}} / S_{L}$ for the isosurface at $c=0.8$. Variation of the correlation coefficients for all cases on the different $c$ isosurfaces among (b) $S_{d}$ and $a_{T}$, (c) $S_{r}$ and $a_{T}$, (d) $S_{n}$ and $a_{T}$, (e) $\left(S_{r}+S_{n}\right)$ and $a_{T}$ and (f) $S_{t}$ and $a_{T}$. 
(negative) correlation between $S_{n}$ and $a_{T}$ on the reactant (product) side. This behaviour can be seen in the correlation coefficients plotted in Figure 11(d), which also indicates that the correlation coefficient between $S_{n}$ and $a_{T}$ does not show any monotonic trend with changes in either $\mathrm{Da}$ or Ka. However, it has been found that the correlation between $S_{n}$ and $a_{T}$ in general remains stronger for smaller values of $u^{\prime}$ when either $\mathrm{Da}$ or $\mathrm{Ka}$ is held constant.

Since $S_{r}$ remains negligible towards the reactant side of the flame brush and $S_{n}$ is positively correlated with $a_{T}$, the correlation coefficient between $\left(S_{r}+S_{n}\right)$ and $a_{T}$ remains positive towards the reactant side (see Figure 11(e)). By contrast, both $S_{r}$ and $S_{n}$ are negatively correlated with $a_{T}$ towards the product side, which leads to a locally negative correlation between $\left(S_{r}+S_{n}\right)$ and $a_{T}$.

Since $a_{T}$ and $\kappa_{m}$ are negatively correlated, the tangential diffusion component $S_{t}$ is positively correlated with $a_{T}$ because $S_{t}$ is proportional to the negative of curvature (i.e., $\left.S_{t}=-2 D \kappa_{m}\right)$. This positive correlation is evident from Figure 11(f), in which the correlation coefficient between $S_{t}$ and $a_{T}$ is shown for isosurfaces of $c$ across the flame brush. The negative correlation between $a_{T}$ and $\kappa_{m}$ (see Figure $7(\mathrm{a})$ ) arises principally due to heat release, and thus, the strength of this correlation weakens with increasing Karlovitz number, since the effects of turbulent velocity fluctuations are likely to mask the heat release effects as the combustion tends more towards the broken reaction zones regime [1]. Moreover, with decreasing Damköhler number the effects of heat release weaken in comparison to those of turbulent straining, as suggested by (11b). As a result of this, the strength of the negative correlation between $a_{T}$ and $\kappa_{m}$ weakens with decreasing (increasing) Damköhler number (Karlovitz number) when $\mathrm{Ka}(\mathrm{Da})$ is held constant (see Figure $7(\mathrm{a})$ ), and this gives rise to a corresponding change in the correlation between $S_{t}$ and $a_{T}$ (see Figure 11(f)). This essentially suggests that the strength of the positive correlation between $S_{t}$ and $a_{T}$ decreases with increasing turbulent Reynolds number $\mathrm{Re}_{t}$ when either $\mathrm{Ka}$ or $\mathrm{Da}$ is held constant. The effects of the positive $S_{t}-a_{T}$ and negative $a_{T}-\kappa_{m}$ correlations remain strongest in the reaction zone and the strengths of these correlations decrease towards both the reactant and product sides of the flame brush (see Figures 11(f) and 7(b), resp.).

It is evident from Figures 11(e) and 11(f) that the positive correlations between $\left(S_{r}+S_{n}\right)$ and $a_{T}$, and between $S_{t}$ and $a_{T}$, aid each other towards the reactant side of the flame brush. By contrast, towards the product side the positive correlation between $S_{t}$ and $a_{T}$ overcomes the negative correlation between $\left(S_{r}+S_{n}\right)$ and $a_{T}$ to result in a net positive correlation between $S_{d}$ and $a_{T}$ in all the cases considered here (see Figure 11(a)). The weakening of the correlation between $S_{t}$ and $a_{T}$ with increasing $\mathrm{Re}_{t}$ ultimately leads to decrease in the strength of the correlation between $S_{d}$ and $a_{T}$ when either $\mathrm{Da}$ or Ka is held constant (see Figures 11(a) and 11(f)).

4.6. Implications for FSD Modelling. In the context of Reynolds Averaged Navier Stokes (RANS) modelling, the generalised FSD $\Sigma_{\text {gen }}$ is defined as [39]

$$
\Sigma_{\text {gen }}=\langle|\nabla c|\rangle,
$$

where $\langle Q\rangle$ indicates the Reynolds averaged value of a general quantity $Q$. It has been demonstrated in $[15,23,26,27]$ that the statistical behaviour of $|\nabla c|$ and the terms of its transport (7) in the thin reaction zones regime depend significantly on the choice of progress variable isosurface $c=c^{*}$. Hence, it is more appropriate to use the generalised FSD (see(12a)) which does not depend on the choice of isosurface. Reynolds averaging of (7) yields the transport equation for the generalised FSD $[2,23,24]$

$$
\begin{aligned}
\frac{\partial \Sigma_{\text {gen }}}{\partial t}+\frac{\partial\left\langle u_{j}\right\rangle_{s} \Sigma_{\text {gen }}}{\partial x_{j}}= & \left\langle\left(\delta_{i j}-N_{i} N_{j}\right) \frac{\partial u_{i}}{\partial x_{j}}\right\rangle_{s} \Sigma_{\text {gen }} \\
& +2\left\langle S_{d} \kappa_{m}\right\rangle_{s} \Sigma_{\text {gen }}-\frac{\partial\left\langle S_{d} N_{i}\right\rangle_{s} \Sigma_{\text {gen }}}{\partial x_{i}}
\end{aligned}
$$

where $\langle Q\rangle_{s}=\langle Q|\nabla c|\rangle /\langle|\nabla c|\rangle$ indicates a surface averaging operation for a general quantity $Q$ [39]. The first term on the left hand side is the transient term while the second term is the advection term. The three terms on the right hand side represent the effects of strain rate, curvature, and propagation, respectively. It is evident from (12b) that the statistical behaviour of the curvature and propagation terms depends on the statistics of displacement speed. These terms can be rewritten in terms of the displacement speed components as

$$
\begin{aligned}
2\left\langle S_{d} \kappa_{m}\right\rangle_{s} \Sigma_{\text {gen }}= & 2\left\langle\left(S_{r}+S_{n}\right) \kappa_{m}\right\rangle_{s} \Sigma_{\text {gen }} \\
& -4\left\langle D \kappa_{m}^{2}\right\rangle_{s} \Sigma_{\text {gen }}, \\
-\nabla \cdot\left\langle S_{d} \vec{N}\right\rangle_{s} \sum_{\text {gen }}= & -\nabla \cdot\left\langle\left(S_{r}+S_{n}\right) \vec{N}\right\rangle_{s} \sum_{\text {gen }} \\
& +2 \nabla \cdot\left\langle D \kappa_{m} \vec{N}\right\rangle_{s} \sum_{\text {gen }} .
\end{aligned}
$$

The variations of all of the terms appearing in these equations with Favre averaged reaction progress variable $\tilde{c}=\langle\rho c\rangle /\langle\rho\rangle$ are shown in Figure 12. The Reynolds averages are evaluated by ensemble averaging the relevant quantities in the directions normal to the direction of mean flame propagation.

It can be seen from Figure 12 that the FSD curvature term $\left\langle S_{d} \nabla \cdot \vec{N}\right\rangle_{s} \sum_{\text {gen }}$ (line with tick symbols) assumes mostly negative value throughout the flame brush for all cases although small positive contributions are found towards the reactant side of the flame brush for small Reynolds numbers (i.e., cases $\mathrm{A}$ and $\mathrm{B})$. The magnitude of the term $2\left\langle\left(S_{r}+S_{n}\right) \kappa_{m}\right\rangle_{s} \Sigma_{\text {gen }}$ (line with filled triangles) remains comparable to the contribution of the term $-4\left\langle D \kappa_{m}^{2}\right\rangle_{s} \Sigma_{\text {gen }}$ (line with open squares) for small values of $\operatorname{Re}_{t}$ (i.e., cases A and B). However, the contribution of $2\left\langle\left(S_{r}+S_{n}\right) \kappa_{m}\right\rangle_{s} \Sigma_{\text {gen }}$ remains smaller than that of $-4\left\langle D \kappa_{m}^{2}\right\rangle_{s} \Sigma_{\text {gen }}$ for larger values of $\mathrm{Re}_{t}$ and the statistical behaviour of the FSD curvature term is then principally governed by the latter contribution. It is also evident from Figure 12 that the magnitude of $\left(-4\left\langle D \kappa_{m}^{2}\right\rangle_{s} \Sigma_{\text {gen }}\right)$ becomes increasingly more important than the magnitude of $2\left\langle\left(S_{r}+S_{n}\right) \kappa_{m}\right\rangle_{s} \Sigma_{\text {gen }}$ with increasing Karlovitz number (Damköhler number) 


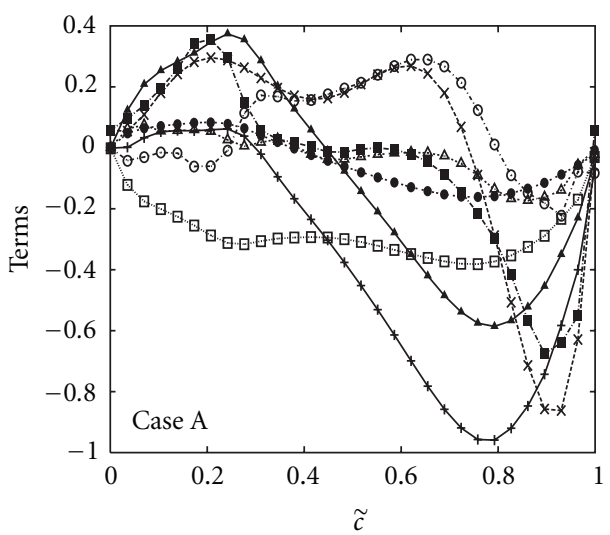

(a)

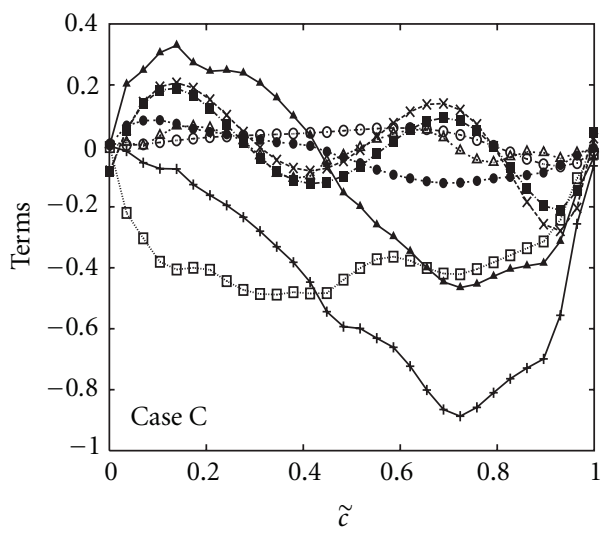

(c)

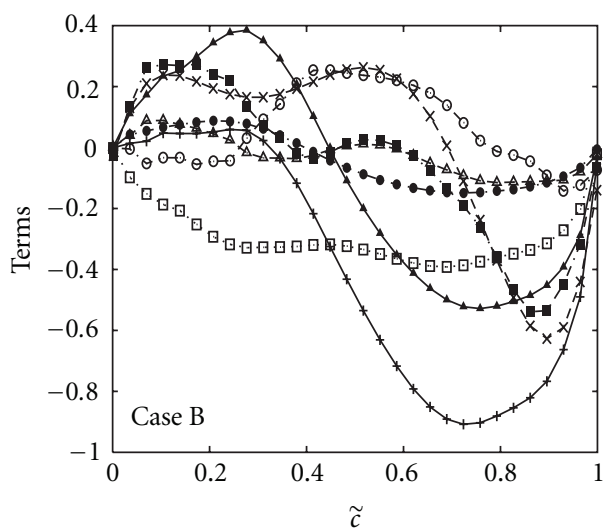

(b)

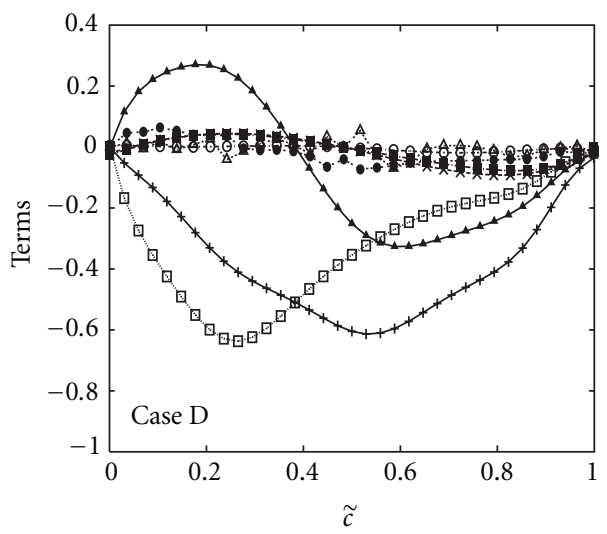

(d)

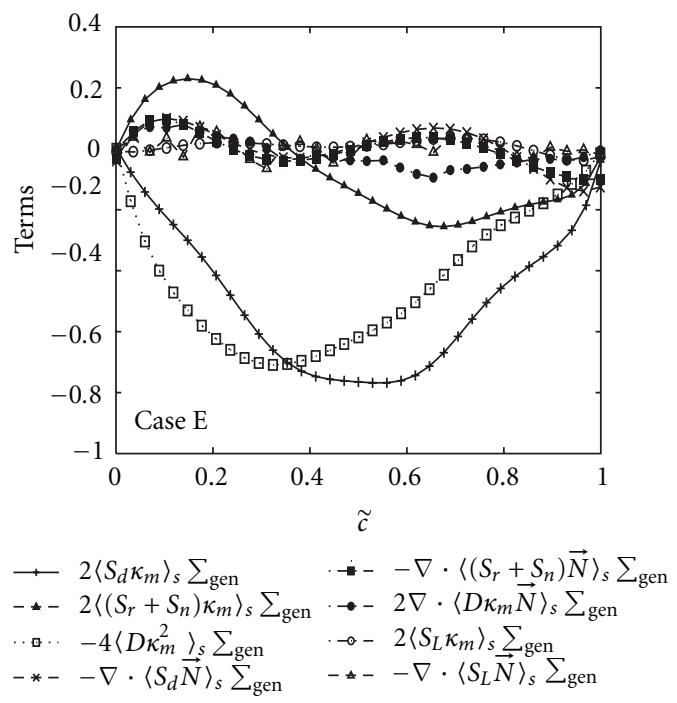

(e)

FIGURE 12: Variation across the flame brush with Favre averaged reaction progress variable $\tilde{c}=\langle\rho c\rangle /\langle\rho\rangle$ of the FSD curvature term $2\left\langle S_{d} \kappa_{m}\right\rangle_{s} \Sigma_{\text {gen }}$ and its contributions (see (12c)) $2\left\langle\left(S_{r}+S_{n}\right) \kappa_{m}\right\rangle_{s} \Sigma_{\text {gen }}$ and $-4\left\langle D \kappa_{m}^{2}\right\rangle_{s} \Sigma_{\text {gen }}$; the FSD propagation term $-\nabla \cdot\left\langle S_{d} \vec{N}\right\rangle_{s} \Sigma_{\text {gen }}$ and its contributions (see (12d)) $-\nabla \cdot\left\langle\left(S_{r}+S_{n}\right) \vec{N}\right\rangle_{s} \Sigma_{\text {gen }}$ and $2 \nabla \cdot\left\langle D \kappa_{m} \vec{N}\right\rangle_{s} \Sigma_{\text {gen }}$; together with the modelled curvature term $2\left\langle S_{L} \kappa_{m}\right\rangle_{s} \Sigma_{\text {gen }}$ and propagation term $-\nabla \cdot\left\langle S_{L} \vec{N}\right\rangle_{s} \Sigma_{\text {gen }}$ for: (a) case A; (b) case B; (c) case C; (d) case D; (e) case E. All the terms are normalised with respect to $S_{L} / \delta_{\mathrm{th}}^{2}$ of the respective cases. 
when $\mathrm{Da}(\mathrm{Ka})$ is held constant. The statistical behaviour of $2\left\langle\left(S_{r}+S_{n}\right) \kappa_{m}\right\rangle_{s} \Sigma_{\text {gen }}$ is determined by the correlation between $|\nabla c|$ and $\kappa_{m}$ and between $\left(S_{r}+S_{n}\right)$ and $\kappa_{m}$ and the strength of both of these correlations tends to weaken with increasing (increasing) Karlovitz number (Damköhler number) when $\mathrm{Da}(\mathrm{Ka})$ is held constant (see Figures 6(b) and $10(\mathrm{~d})$ ), causing a corresponding reduction in the relative magnitude of the term $2\left\langle\left(S_{r}+S_{n}\right) \kappa_{m}\right\rangle_{s} \Sigma_{\text {gen }}$.

The FSD propagation term $-\nabla \cdot\left\langle S_{d} \vec{N}\right\rangle_{s} \Sigma_{\text {gen }}$ (line with crosses) remains positive towards the reactant side, but it eventually becomes negative towards the product side. It can be seen that the term $-\nabla \cdot\left\langle\left(S_{r}+S_{n}\right) \vec{N}\right\rangle_{s} \Sigma_{\text {gen }}$ (line with filled squares) remains almost equal to the FSD propagation term itself, because the contribution of $2 \nabla \cdot\left\langle D \kappa_{m} \vec{N}\right\rangle_{s} \Sigma_{\text {gen }}$ (line with open circles) remains negligible throughout the flame brush due to the statistically planar nature of the flames considered here. The contribution of $2\left\langle\left(S_{r}+S_{n}\right) \kappa_{m}\right\rangle_{s} \Sigma_{\text {gen }}$ remains positive towards the reactant side and assumes negative values towards the product side.

In some previous studies, the FSD curvature and propagation terms have been modelled by replacing $S_{d}$ with $S_{L}$ to yield the modelled expressions $2\left\langle S_{L} \kappa_{m}\right\rangle_{s} \Sigma_{\text {gen }}$ and $-\nabla \cdot\left\langle S_{L} \vec{N}\right\rangle_{s} \Sigma_{\text {gen }}$, respectively; [40, 41]. Figure 12 further indicates that there are significant differences between the original terms and these modelled expressions, which are shown, respectively, by a line with filled circles and a line with open triangles. This essentially indicates that $S_{d}$ cannot be approximated simply by $S_{L}$ in the modelling of the FSD curvature and propagation terms. Moreover, Figure 12 suggests that modelling of $\left\langle\kappa_{m}^{2}\right\rangle_{s}$ is necessary for the closure of the FSD curvature term especially in the thin reaction zones regime since the term $-4\left\langle D \kappa_{m}^{2}\right\rangle_{s} \Sigma_{\text {gen }}$ remains close to $2\left\langle S_{d} \kappa_{m}\right\rangle_{s} \sum_{\text {gen }}$ throughout the flame brush for high values of the turbulent Reynolds number. The effects of turbulent Reynolds number on the modelling of the FSD curvature and propagation terms are beyond of the scope of this study and will be addressed in future work.

\section{Conclusions}

The turbulent Reynolds number dependence of the statistics of displacement speed in turbulent premixed flames has been studied using a DNS database of statistically planar flames, in which the variation of turbulent Reynolds number $\mathrm{Re}_{t}$ from 20 to 100 has been brought about by modifying either Damköhler number Da or Karlovitz number Ka independently of each other. As both $\mathrm{Da}$ and Ka are functions of $u^{\prime} / S_{L}$ and $l / \delta_{\mathrm{th}}$, the variation of $\mathrm{Re}_{t}$ for the present database has been achieved by modifying the initial values of $u^{\prime} / S_{L}$ and $l / \delta_{\text {th }}$ simultaneously.

The mean value of displacement speed remains positive throughout the flame brush, but there is nonzero probability of finding negative values of displacement speed, in accordance with the findings of previous work. It has been shown here that the probability of finding negative displacement speed increases with increasing turbulent Reynolds number when the Damköhler number is held constant.
The dependences of tangential strain rate and dilatation rate on flame curvature are shown to have a significant influence on the strain rate and curvature dependences of the SDF $|\nabla c|$, which in turn affects the statistical behaviour of displacement speed in response to strain rate and curvature. It has been found that the variation of turbulent Reynolds number does not alter the qualitative nature of the correlations between tangential strain rate and dilatation rate with curvature, but the strength of these correlations is found to weaken with increasing turbulent Reynolds number when either Damköhler or Karlovitz number is held constant. Similarly, the strain rate and curvature dependences of displacement speed are shown to weaken with increasing turbulent Reynolds number when either Damköhler or Karlovitz number is kept unaltered.

Detailed physical explanations are provided to explain the influences of turbulent Reynolds number on the strain rate and curvature dependences of displacement speed in terms of the individual response of the reaction, normal diffusion and tangential diffusion components of displacement speed.

The implications of the turbulent Reynolds number dependence of displacement speed for the modelling of the FSD transport equation has been explored. The statistical behaviour of the FSD curvature term contributions arising due to the combined reaction and normal diffusion components of displacement speed and to the tangential diffusion component of displacement speed have been analysed in detail. At low turbulence Reynolds numbers, the magnitudes of these contributions remain comparable. As the turbulence Reynolds number increases, the curvature dependence of the tangential component of displacement speed ensures that this contribution becomes increasingly important and is the predominant influence on the FSD curvature term for larger values of turbulent Reynolds number. Hence, the curvature dependence of the tangential diffusion component of displacement speed cannot be ignored for accurate modelling of the FSD curvature term for flames within the thin reaction zones regime.

It is worth noting that the effects of differential diffusion of heat and mass are not taken into account in the present study. However, some previous DNS studies with detailed chemistry $[3-7,9]$ suggested that intermediate species may play an important role even for the flames with global Lewis number close to unity. Moreover, only a moderate range of turbulent Reynolds number has been considered in this study, so three-dimensional DNS studies with detailed chemistry and at higher values of turbulent Reynolds number will be needed for deeper understanding and for the purpose of quantitative predictions.

\section{Acknowledgment}

N. Chakraborty gratefully acknowledges the financial assistance of the EPSRC, UK. 


\section{References}

[1] N. Peters, Turbulent Combustion, Cambridge University Press, Cambridge, UK, 2000.

[2] S. M. Candel and T. J. Poinsot, "Flame stretch and the balance equation for the flame area," Combustion Science and Technology, vol. 70, pp. 1-15, 1988.

[3] T. Echekki and J. H. Chen, "Unsteady strain rate and curvature effects in turbulent premixed methane-air flames," Combustion and Flame, vol. 106, no. 1-2, pp. 184-202, 1996.

[4] I. R. Gran, T. Echekki, and J. H. Chen, "Negative flame speed in an unsteady 2-D premixed flame: a computational study," Proceedings of the Combustion Institute, vol. 26, no. 1, pp. 323329, 1996.

[5] J. H. Chen and H. G. Im, "Correlation of flame speed with stretch in turbulent premixed methane/air flames," Proceedings of the Combustion Institute, vol. 27, pp. 819-826, 1998.

[6] N. Peters, P. Terhoeven, J. H. Chen, and T. Echekki, "Statistics of flame displacement speeds from computations of 2-D unsteady methane-air flames," Proceedings of the Combustion Institute, vol. 27, pp. 833-839, 1998.

[7] T. Echekki and J. H. Chen, "Analysis of the contribution of curvature to premixed flame propagation," Combustion and Flame, vol. 118, no. 1-2, pp. 308-311, 1999.

[8] J. H. Chen and H. G. Im, "Stretch effects on the burning velocity of turbulent premixed hydrogen/air flames," Proceedings of the Combustion Institute, vol. 28, no. 1, pp. 211-218, 2000.

[9] H. G. Im and J. H. Chen, "Preferential diffusion effects on the burning rate of interacting turbulent premixed hydrogen-air flames," Combustion and Flame, vol. 126, no. 3, pp. 246-258, 2002.

[10] E. R. Hawkes and J. H. Chen, "Direct numerical simulation of hydrogen-enriched lean premixed methane-air flames," Combustion and Flame, vol. 138, no. 3, pp. 242-258, 2004.

[11] E. R. Hawkes and J. H. Chen, "Evaluation of models for flame stretch due to curvature in the thin reaction zones regime," Proceedings of the Combustion Institute, vol. 30, pp. 647-653, 2005.

[12] E. R. Hawkes and J. H. Chen, "Comparison of direct numerical simulation of lean premixed methane-air flames with strained laminar flame calculations," Combustion and Flame, vol. 144, no. 1-2, pp. 112-125, 2006.

[13] N. Chakraborty, E. R. Hawkes, J. H. Chen, and R. S. Cant, "The effects of strain rate and curvature on surface density function transport in turbulent premixed methane-air and hydrogenair flames: a comparative study," Combustion and Flame, vol. 154, no. 1-2, pp. 259-280, 2008.

[14] N. Chakraborty and S. Cant, "Unsteady effects of strain rate and curvature on turbulent premixed flames in an inflowoutflow configuration," Combustion and Flame, vol. 137, no. 1-2, pp. 129-147, 2004.

[15] N. Chakraborty and R. S. Cant, "Effects of strain rate and curvature on surface density function transport in turbulent premixed flames in the thin reaction zones regime," Physics of Fluids, vol. 17, no. 6, article 65108, pp. 1-15, 2005.

[16] N. Chakraborty and R. S. Cant, "Influence of Lewis number on curvature effects in turbulent premixed flame propagation in the thin reaction zones regime," Physics of Fluids, vol. 17, no. 10, Article ID 105105, pp. 1-20, 2005.

[17] K. W. Jenkins, M. Klein, N. Chakraborty, and R. S. Cant, "Effects of strain rate and curvature on the propagation of a spherical flame kernel in the thin-reaction-zones regime," Combustion and Flame, vol. 145, no. 1-2, pp. 415-434, 2006.
[18] M. Klein, N. Chakraborty, K.W. Jenkins, and R. S. Cant, "Effects of initial radius on the propagation of spherical premixed flame Kernels in turbulent environment," Physics of Fluids, vol. 18, Article ID 055102, pp. 1-15, 2006.

[19] N. Chakraborty and R. S. Cant, "Influence of Lewis number on strain rate effects in turbulent premixed flame propagation," International Journal of Heat and Mass Transfer, vol. 49, no. 13-14, pp. 2158-2172, 2006.

[20] N. Chakraborty, M. Klein, and R. S. Cant, "Stretch rate effects on displacement speed in turbulent premixed flame kernels in the thin reaction zones regime," Proceedings of the Combustion Institute, vol. 31, pp. 1385-1392, 2007.

[21] N. Chakraborty, "Comparison of displacement speed statistics of turbulent premixed flames in the regimes representing combustion in corrugated flamelets and thin reaction zones," Physics of Fluids, vol. 19, no. 10, Article ID 105109, pp. 1-20, 2007.

[22] I. Han and K. Y. Huh, "Roles of displacement speed on evolution of flame surface density for different turbulent intensities and Lewis numbers in turbulent premixed combustion," Combustion and Flame, vol. 152, no. 1-2, pp. 194-205, 2008.

[23] N. Chakraborty and R. S. Cant, "A priori analysis of the curvature and propagation terms of the flame surface density transport equation for large eddy simulation," Physics of Fluids, vol. 19, no. 10, Article ID 105101, pp. 1-22, 2007.

[24] N. Chakraborty and R. S. Cant, "Direct numerical simulation analysis of the flame surface density transport equation in the context of large eddy simulation," Proceedings of the Combustion Institute, vol. 32, pp. 1445-1453, 2009.

[25] W. Kollmann and J. H. Chen, "Pocket formation and the flame surface density equation," Proceedings of the Combustion Institute, vol. 27, pp. 927-934, 1998.

[26] N. Chakraborty and M. Klein, "Influence of Lewis number on the surface density function transport in the thin reaction zone regime for turbulent premixed flames," Physics of Fluids, vol. 20, no. 6, Article ID 065102, 2008.

[27] N. Chakraborty and M. Klein, "Effects of global flame curvature on surface density function transport in turbulent premixed flame kernels in the thin reaction zones regime," Proceedings of the Combustion Institute, vol. 32, pp. 1435-1443, 2009.

[28] K. W. Jenkins and R. S. Cant, "DNS of turbulent flame kernels," in Proceedings of the 2nd AFOSR Conference on DNS and LES, Knight and Sakell, Eds., pp. 192-202, Kluwer Academic Publishers, Rutgers University, 1999.

[29] T. J. Poinsot and S. K. Lele, "Boundary conditions for direct simulations of compressible viscous flows," Journal of Computational Physics, vol. 101, no. 1, pp. 104-129, 1992.

[30] A. A. Wray, "Minimal storage time advancement schemes for spectral methods," Tech. Rep., NASA Ames Research Center, Calif, USA, 1990.

[31] R. S. Rogallo, "Numerical experiments in homogeneous turbulence," NASA Technical Memorandum 81315, NASA Ames Research Center, Calif, USA, 1981.

[32] T. Poinsot, T. Echekki, and M. Mungal, "A study of the laminar flame tip and implications for turbulent premixed combustion," Combustion Science and Technology, vol. 81, no. 1-3, pp. 45-73, 1992.

[33] D. S. Louch and K. N. C. Bray, "Vorticity in unsteady premixed flames: vortex pair-premixed flame interactions under imposed body forces and various degrees of heat release and laminar flame thickness," Combustion and Flame, vol. 125, no. 4, pp. 1279-1309, 2001. 
[34] T. C. Treurniet, F. T. M. Nieuwstadt, and B. J. Boersma, "Direct numerical simulation of homogeneous turbulence in combination with premixed combustion at low Mach number modelled by the G-equation," Journal of Fluid Mechanics, vol. 565 , pp. 25-62, 2006.

[35] H. Kobayashi, T. Kawahata, K. Seyama, T. Fujimari, and J. S. Kim, "Relationship between the smallest scale of flame wrinkles and turbulence characteristics of high-pressure, high-temperature turbulent premixed flames," Proceedings of the Combustion Institute, vol. 29, pp. 1793-1799, 2002.

[36] N. Chakraborty, G. Hartung, M. Katragadda, and C. F. Kaminski, "A numerical comparison of 2D and 3D densityweighted displacement speed statistics and implications for laser based measurements of flame displacement speed," Combustion and Flame, vol. 158, no. 7, pp. 1372-1390, 2011.

[37] C. Meneveau and T. Poinsot, "Stretching and quenching of flamelets in premixed turbulent combustion," Combustion and Flame, vol. 86, no. 4, pp. 311-332, 1991.

[38] G. Joulin, "On the response of premixed flames to timedependent stretch and curvature," Combustion Science and Technology, vol. 97, no. 1-3, pp. 219-229, 1994.

[39] M. Boger, D. Veynante, H. Boughanem, and A. Trouve, "Direct numerical simulation analysis of flame surface density concept for large eddy simulation of turbulent premixed combustion," Proceedings of the Combustion Institute, vol. 27, pp. 917-925, 1998.

[40] D. Veynante, J. M. Duclos, and J. Piana, "Experimental analysis of flamelet models for premixed turbulent combustion," Proceedings of the Combustion Institute, vol. 25, no. 1, pp. 1249-1256, 1994.

[41] D. Veynante, J. Piana, J. M. Duclos, and C. Martel, "Experimental analysis of flame surface density models for premixed turbulent combustion," Proceedings of the Combustion Institute, vol. 26, no. 1, pp. 413-420, 1996. 

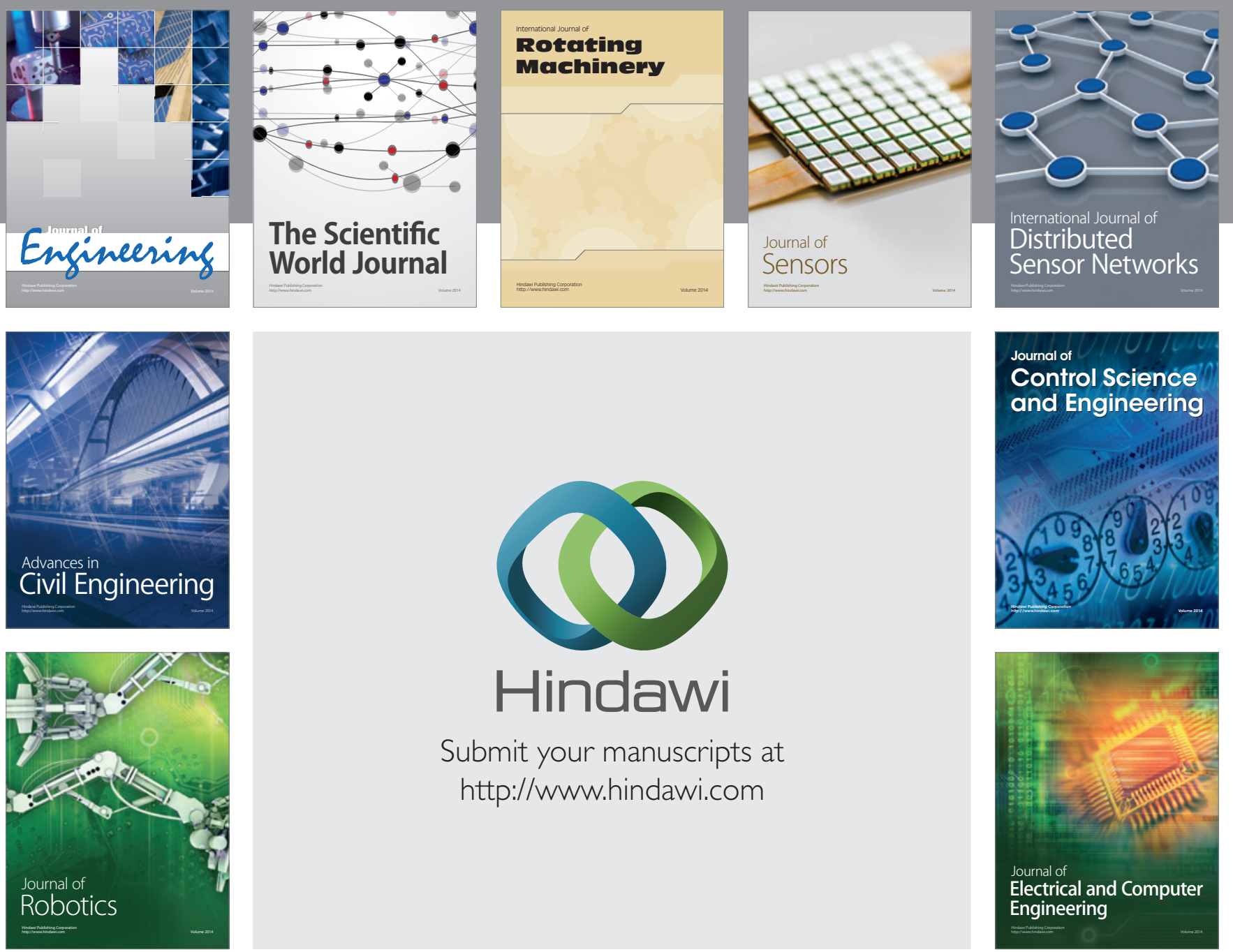

Submit your manuscripts at

http://www.hindawi.com
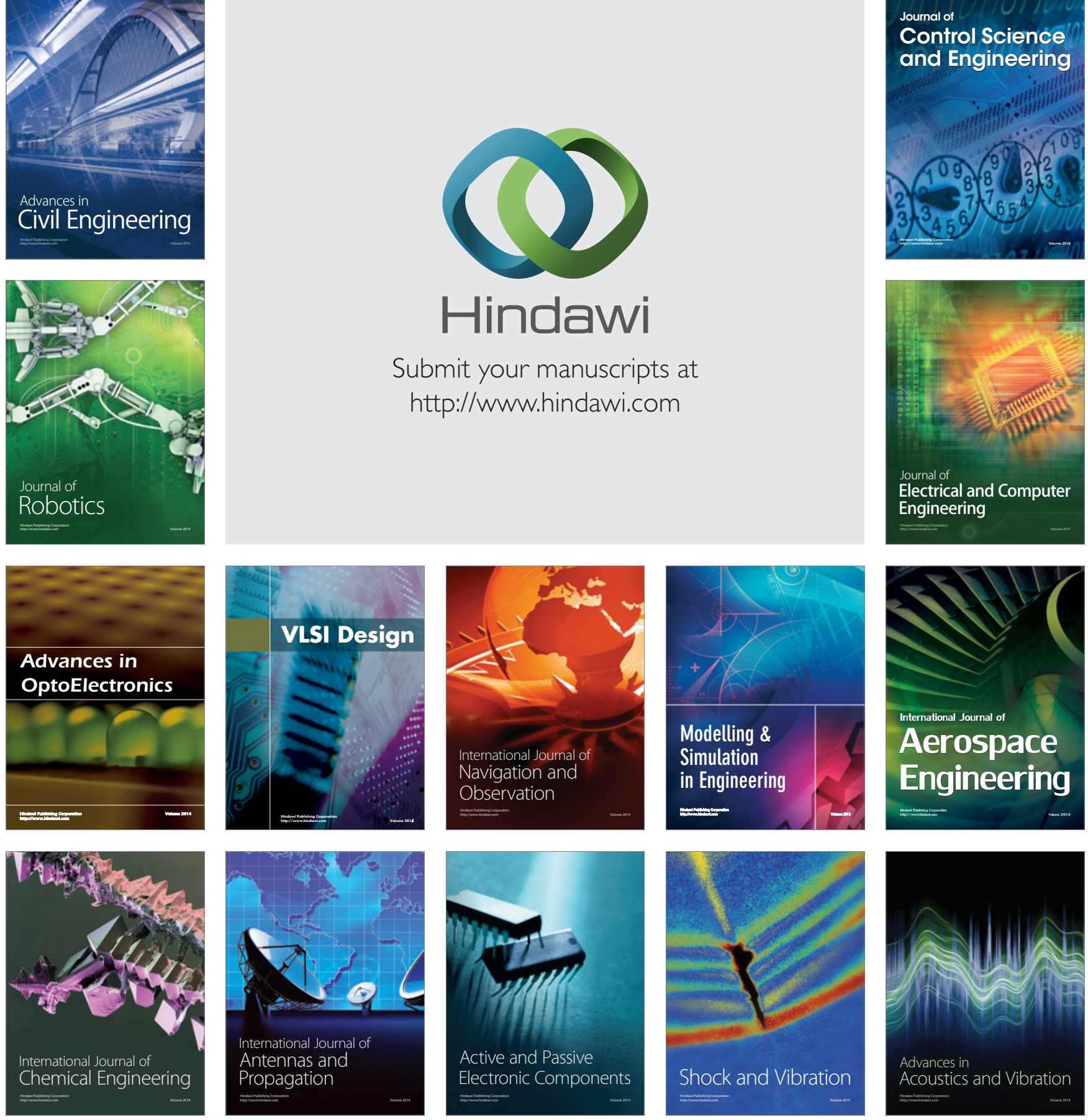